\title{
Classification of Invariant Star Products up to Equivariant Morita Equivalence on Symplectic Manifolds
}

\author{
Stefan Jansen, Nikolai Neumaier, Gregor Schaumann, Stefan Waldmann* \\ Fakultät für Mathematik und Physik \\ Albert-Ludwigs-Universität Freiburg \\ Physikalisches Institut \\ Hermann-Herder-Straße 3 \\ D 79104 Freiburg \\ Germany
}

April 2010

\begin{abstract}
In this paper we investigate equivariant Morita theory for algebras with momentum maps and compute the equivariant Picard groupoid in terms of the Picard groupoid explicitly. We consider three types of Morita theory: ring-theoretic equivalence, ${ }^{*}$-equivalence and strong equivalence. Then we apply these general considerations to star product algebras over symplectic manifolds with a Lie algebra symmetry. We obtain the full classification up to equivariant Morita equivalence.
\end{abstract}

${ }^{*}$ Corresponding author: Stefan.Waldmann@physik.uni-freiburg.de 


\section{Contents}

1 Introduction $\quad 2$

2 Preliminaries on equivariant Morita theory 4

2.1 Picard groupoids . . . . . . . . . . . . . . . . . 4

2.2 Equivariant Picard groupoids . . . . . . . . . . . . . . . 5

2.3 The groups $\mathrm{Gl}(H, \mathcal{A})$ and $\mathrm{U}(H, \mathcal{A}) \ldots \ldots \ldots \ldots$

3 Fedosov star products and quantum momentum maps 8

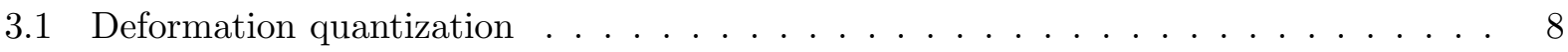

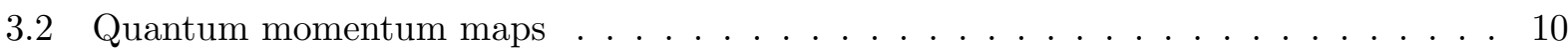

3.3 Equivariant cohomology . . . . . . . . . . . . . . . . . 12

4 Momentum maps and the splitting 13

4.1 Momentum maps . . . . . . . . . . . . . . . . . . . . . . . . 14

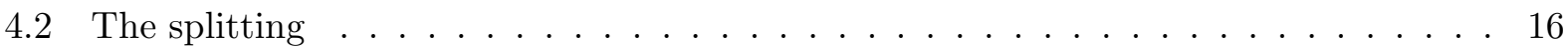

5 Equivariant Morita equivalence of star products $\quad 21$

5.1 Classical and semiclassical limits of a $\mathfrak{g}$-equivariant equivalence bimodule . . . . . . 21

5.2 Characterization of equivariant Morita equivalent star products . . . . . . . . . . . 24

5.3 The role of quantum momentum maps . . . . . . . . . . . . . . 26

\section{Introduction}

The aim of this paper is two-fold: on the one hand we continue the study of equivariant Morita theory of algebras with respect to Hopf algebra actions as initiated in [16]. On the other hand, we consider the particular case of star product algebras over symplectic manifolds with symmetries coming from Lie algebra actions on the underlying manifold.

On the general algebraic side, the algebras in question carry a left action of a fixed Hopf algebra $H$. We are interested in the groupoid of all isomorphism classes of Morita equivalence bimodules with compatible action of $H$. Beside this purely algebraic case, we are interested in the situation that the algebras are defined over a ring $C=R(i)$ with an ordered $\operatorname{ring} R$ and $i^{2}=-1$ such that they carry a *-involution. In this case, also the Hopf algebra is required to be a Hopf *-algebra and the action is required to be $\mathrm{a}^{*}$-action. Moreover, for the equivalence bimodules one demands algebra-valued inner products either with or without complete positivity. This gives the three flavours of Morita theory we are interested in: ring-theoretic Morita theory, ${ }^{*}$-Morita theory, and strong Morita theory. We base our study of Morita theory on the corresponding Picard groupoids. While in [16] the general interplay of $H$-equivariance with these three types of Morita theory was studied, we focus on a more particular situation where the action of $H$ allows a momentum map, i.e. an algebra homomorphism $J \longrightarrow \mathcal{A}$ which induces the action as an inner action. In this case, the in general very difficult question whether Morita equivalence implies also equivariant Morita equivalence can be answered positively in a trivial way. Even more precisely, we obtain that the canonical groupoid morphisms of forgetting the equivariance

$$
\mathrm{Pic}_{H} \longrightarrow \mathrm{Pic}, \quad \mathrm{Pic}_{H}^{*} \longrightarrow \mathrm{Pic}^{*}, \quad \text { and } \mathrm{Pic}_{H}^{\text {str }} \longrightarrow \mathrm{Pic}^{\text {str }}
$$

are not only surjective but possess a canonical right inverse constructed from the momentum maps. The kernels of (1.1) for all three flavours of Morita theory were studied in detail in [16]. 
Moreover, we show that the existence of a momentum map is an invariant under $H$-equivariant Morita equivalence. Thus the restriction of the Picard groupoids to this particular class of actions and algebras is reasonable as we would not leave these connected components of the groupoids anyway.

On the star product side we consider $\mathfrak{g}$-invariant star products on symplectic manifolds with a symplectic action of a finite-dimensional Lie algebra $\mathfrak{g}$. The action may or may not arise from a corresponding Lie group action. The only technical condition we need throughout is that the action preserves a connection, and consequently also preserves a symplectic torsion-free connection. In this case, one has strong existence and classification results of such $\mathfrak{g}$-invariant star products according to [4] by a $\mathfrak{g}$-invariant characteristic class $c^{\mathfrak{g}}(\cdot)$ in the formal series in the $\mathfrak{g}$-invariant second deRham cohomology of $M$. A particularly nice situation is obtained if in addition the classical $\mathfrak{g}$-action allows for a classical momentum map. Then also the star products which allow a quantum momentum map have been studied and classified according to [14,20]. We show that the notion of quantum momentum maps is equivalent to the momentum maps in the above Hopf algebraic approach to equivariant Morita theory if the Hopf algebra is chosen to be a "rescaled" version of the universal enveloping algebra of $\mathfrak{g}$.

For general star products on symplectic manifolds the classification up to Morita equivalence (and also up to *-Morita equivalence and strong Morita equivalence) is available from [7]. Thus the task is to combine both classification results to get the classification of $\mathfrak{g}$-invariant star products up to $\mathfrak{g}$-equivariant Morita equivalence. Here we obtain the main result of this paper:

Theorem 1.1 Suppose there exists a $\mathfrak{g}$-invariant connection on a symplectic manifold $M$ and let $\star$ and $\star^{\prime}$ be two $\mathfrak{g}$-invariant (Hermitian) star products. Then $\star$ and $\star^{\prime}$ are $\mathfrak{g}$-equivariantly (strongly) Morita equivalent if and only if there is a $\mathfrak{g}$-equivariant symplectomorphism $\Psi$ such that $\Psi^{*} c^{\mathfrak{g}}\left(\star^{\prime}\right)-c^{\mathfrak{g}}(\star)$ is in the image of the first map in

$$
\mathrm{H}_{\mathfrak{g}}^{2}(M, \mathbb{C}) \longrightarrow \mathrm{H}_{\mathrm{dR}}^{2}(M, \mathbb{C})^{\mathfrak{g}} \longrightarrow \mathrm{H}_{\mathrm{dR}}^{2}(M, \mathbb{C}),
$$

and maps to a $2 \pi \mathrm{i}$-integral deRham cohomology class under the second map.

The proof of this theorem is based on a careful analysis of the semiclassical limit of $\mathfrak{g}$-equivariant equivalence bimodules on one hand and the invariant Fedosov construction on the other hand. For star products with quantum momentum maps the proof simplifies in so far as the problem of lifting the Lie algebra action from the algebras to the bimodule is trivial thanks to the surjectivity of (1.1). This brings together both parts of the paper.

Let us finally mention several open questions and further possible developments: On one hand, the case of Poisson manifolds is clearly challenging. Here one can expect that things go more or less the same way. However, it will require more advanced technology: first a classification of invariant star products has to be achieved. Then the characterization of Morita equivalent star products [6,17] has to be cast into the equivariant setting. For both steps the equivariant formality theorems based on the existence of a $\mathfrak{g}$-invariant connection [10] should be sufficient. On the other hand, one can pass to higher rank bundles and hence to endomorphism algebras $\Gamma^{\infty}(\operatorname{End}(E))$ and their deformations. This would eventually lead to a notion of equivariant cohomology with values in endomorphism bundles. From our general surjectivity statements in (1.1) one can expect to get also here some nice applications for the geometric lifting problems.

The paper is organised as follows: in Section 2 we recall the basic definitions from equivariant Morita theory and explain the kernels of the groupoid morphisms (1.1). Section 3 contains background information on deformation quantization, in particular on the $\mathfrak{g}$-invariant star products and their classification where we also reformulate the well-known criteria on the existence of quantum 
momentum maps in terms of equivariant cohomology. In Section 4 we discuss the general algebraic framework of equivariant Morita theory for algebras with momentum maps and we proof the first main result of this paper, the surjectivity of (1.1), in Theorem 4.7. Finally, Section 5 contains the discussion of the semiclassical limit of equivariant equivalence bimodules of star products as well as the proof of Theorem 1.1 both in the general case and with quantum momentum maps.

\section{Preliminaries on equivariant Morita theory}

We briefly recall the most important notions of equivariant Morita equivalence for unital rings and for *-algebras over ordered rings essentially following [16]. As a first remark we note that the terms "covariant" and "equivariant" are used synonymously in this context.

\subsection{Picard groupoids}

In the following, we will meet three types of Morita theory: the original one based on rings alone and two more specific notions taking into account additional structures on the rings in question. In particular, we are interested in ${ }^{*}$-algebras over rings $C$ where $C=R(i)$ is a ring extension of an ordered ring $\mathrm{R}$ by a square root $\mathrm{i}$ of -1 , see $[9$ for a detailed discussion. The main example for us, beside $\mathbb{R}$ and $\mathbb{C}$, will be the ring $\mathbb{R}[[\lambda]]$ of real formal power series with its natural ordering and the corresponding $\mathbb{C}[[\lambda]]$.

In the case of *-algebras the theory works well if we add the requirement that all algebras are idempotent and non-degenerate. However, to keep things simple, we assume that all rings and algebras are unital throughout this work.

We base our approach to Morita theory on "invertible bimodules". A $(\mathcal{B}, \mathcal{A})$-bimodule ${ }_{\mathcal{B}} \mathcal{E}_{\mathcal{A}}$ is called invertible if one can find another bimodule ${ }_{\mathcal{A}} \widetilde{\mathcal{E}}_{\mathcal{B}}$ such that ${ }_{\mathcal{B}} \mathcal{E}_{\mathcal{A}} \otimes_{\mathcal{A}} \widetilde{\mathcal{E}}_{\mathcal{B}} \cong{ }_{\mathcal{B}} \mathcal{B}_{\mathcal{B}}$ and ${ }_{\mathcal{A}} \widetilde{\mathcal{E}}_{\mathcal{B}} \otimes_{\mathcal{B}} \mathcal{E}_{\mathcal{A}} \cong{ }_{\mathcal{A}} \mathcal{A}_{\mathcal{A}}$ as bimodules. In fact, one considers isomorphism classes of $(\mathcal{B}, \mathcal{A})$-bimodules as generalized arrows from $\mathcal{A}$ to $\mathcal{B}$ with composition given by the tensor product and units given by the isomorphism classes of the canonical bimodules ${ }_{\mathcal{A}} \mathcal{A}_{\mathcal{A}}$. As we are dealing with unital rings and algebras throughout, we require $\mathbb{1}_{\mathcal{B}} \cdot x=x=x \cdot \mathbb{1}_{\mathcal{A}}$ for all the elements $x$ in every $(\mathcal{B}, \mathcal{A})$-bimodule. This way, one obtains the category Bimod and the invertible bimodules correspond precisely to the invertible morphisms in this category which constitute the Picard groupoid Pic. Strictly speaking, one should fix a suitable Grothendieck universe to work within. We will assume that this issue has been settled. It is a classical theorem of Morita that invertible bimodules are characterized to be finitely generated full projective right $\mathcal{A}$-modules with $\mathcal{B} \cong \operatorname{End}_{\mathcal{A}}\left(\mathcal{E}_{\mathcal{A}}\right)$. Invertible bimodules are also called equivalence bimodules and rings allowing for an equivalence bimodule are called Morita equivalent.

In the case of *-algebras we require more structure on the bimodules: first we always assume to have a compatible $\mathrm{C}$-module structure on all occurring bimodules. For ${ }_{\mathcal{B}} \mathcal{E}_{\mathcal{A}}$ we require an $\mathcal{A}$-valued inner product, i.e. a map $\langle\cdot, \cdot\rangle_{\mathcal{A}}:{ }_{B} \mathcal{E}_{\mathcal{A}} \times_{\mathcal{B}} \mathcal{E}_{\mathcal{A}} \longrightarrow \mathcal{A}$ which is $\mathrm{C}$-linear and $\mathcal{A}$-linear to the right in the second argument, obeys $\left(\langle x, y\rangle_{\mathcal{A}}\right)^{*}=\langle y, x\rangle_{\mathcal{A}}$ for all $x, y \in{ }_{\mathcal{B}} \mathcal{E}_{\mathcal{A}}$, and which is non-degenerate in the usual sense that $\langle x, y\rangle_{\mathcal{A}}=0$ for all $y$ implies $x=0$. Finally, the left multiplications with elements $b \in \mathcal{B}$ should satisfy $\langle b \cdot x, y\rangle_{\mathcal{A}}=\left\langle x, b^{*} \cdot y\right\rangle_{\mathcal{A}}$. In particular, the left multiplications are adjointable. Such bimodules are now called inner product bimodules. Morphisms between such bimodules are $(\mathcal{B}, \mathcal{A})$-bimodule morphisms which are adjointable. In this context, isomorphisms always refer to isometric (and hence adjointable) isomorphisms with respect to the inner product.

The canonical bimodule ${ }_{\mathcal{A}} \mathcal{A}_{\mathcal{A}}$ carries the canonical inner product $\langle a, b\rangle=a^{*} b$. On tensor products we use Rieffel's formula for an inner product: Let ${ }_{e} \mathcal{F}_{\mathcal{B}}$ and ${ }_{\mathcal{B}} \mathcal{E}_{\mathcal{A}}$ be inner product bimodules 
then

$$
\left\langle y \otimes_{\mathcal{B}} x, y^{\prime} \otimes_{\mathcal{B}} x^{\prime}\right\rangle_{\mathcal{A}}^{\mathcal{F} \otimes \mathcal{E}}=\left\langle x,\left\langle y, y^{\prime}\right\rangle_{\mathcal{B}}^{\mathcal{F}} \cdot x^{\prime}\right\rangle_{\mathcal{A}}^{\mathcal{E}}
$$

for $y, y^{\prime} \in e_{e} \mathcal{F}_{\mathcal{B}}$ and $x, x^{\prime} \in{ }_{\mathcal{B}} \mathcal{E}_{\mathcal{A}}$ extends to a well-defined inner product on ${ }_{e} \mathcal{F}_{\mathcal{B}} \otimes_{\mathcal{B}} \mathcal{E}_{\mathcal{B}}$ obeying all requirements except that it may be degenerate. Fortunately, the degeneracy space is a $(\mathcal{C}, \mathcal{A})$ subbimodule. Thus we quotient by it and get a new inner product $(\mathcal{C}, \mathcal{A})$-bimodule denoted by $e^{\mathcal{F}_{\mathcal{B}}} \widehat{\otimes}_{\mathcal{B} B} \mathcal{E}_{\mathcal{A}}$. With the (unital) *-algebras as objects and isomorphism classes of inner product bimodules as morphisms, ${ }_{\mathcal{A}} \mathcal{A}_{\mathcal{A}}$ as units, and the internal tensor product $\widehat{\otimes}_{\mathcal{B}}$ as composition, one obtains the category Bimod* in analogy to Bimod. The invertible bimodules in this category are now called *-equivalence bimodules and two *-algebras allowing for such a *-equivalence bimodule are called *-Morita equivalent, see [1]. The resulting groupoid of invertible arrows in Bimod* is called the *-Picard groupoid Pic*.

Up to now we have not yet used the underlying order structure of $\mathrm{R}$ : Recall that a C-linear functional $\omega: \mathcal{A} \longrightarrow \mathrm{C}$ is called positive if $\omega\left(a^{*} a\right) \geq 0$ for all $a \in \mathcal{A}$ in the sense of $\mathrm{R} \subseteq \mathrm{C}$. An algebra element $a \in \mathcal{A}$ is called positive if for all positive $\omega$ we have $\omega(a) \geq 0$. This allows for the following definition: an inner product $\langle\cdot, \cdot\rangle_{\mathcal{A}}$ is called completely positive if for all $n$ and all $x_{1}, \ldots, x_{n} \in{ }_{\mathcal{B}} \mathcal{E}_{\mathcal{A}}$ the matrix $\left(\left\langle x_{i}, x_{j}\right\rangle_{\mathcal{A}}\right) \in M_{n}(\mathcal{A})$ is positive. In this case, ${ }_{\mathcal{B}} \mathcal{E}_{\mathcal{A}}$ is also called a pre Hilbert $(\mathcal{B}, \mathcal{A})$-bimodule. As a matter of fact, the internal tensor product of two completely positive inner products is again completely positive. This way, one obtains the subcategory of pre Hilbert bimodules Bimod ${ }^{\text {str }}$ of Bimod*. The invertible arrows are now called strong equivalence bimodules and two algebras allowing for a strong equivalence bimodule are called strongly Morita equivalent, see [9]. The invertible arrows constitute now the strong Picard groupoid denoted by Pic ${ }^{\text {str }}$. Finally, we note that for ${ }^{*}$-equivalence bimodules the inner product (2.1) is already non-degenerate. Hence we do not need the quotient procedure here.

The invertible inner product bimodules in Bimod* can now be characterized as follows: they are equivalence bimodules and there is a $\mathcal{B}$-valued inner product ${ }_{\mathcal{B}}\langle\cdot, \cdot\rangle$ with $\mathcal{B}$-linearity properties to the left and compatibility with the right $\mathcal{A}$-multiplications such that both inner products are full in the sense that $\langle\mathcal{E}, \mathcal{E}\rangle_{\mathcal{A}}=\mathcal{A}$ and ${ }_{\mathcal{B}}\langle\mathcal{E}, \mathcal{E}\rangle=\mathcal{B}$, and such that they are compatible in the sense that ${ }_{\mathcal{B}}\langle x, y\rangle \cdot z=x \cdot\langle y, z\rangle_{\mathcal{A}}$ for all $x, y, z \in{ }_{\mathcal{B}} \mathcal{E}_{\mathcal{A}}$. In the strong case, ${ }_{\mathcal{B}}\langle\cdot, \cdot\rangle$ is in addition completely positive as well. One can then show that a candidate for the inverse of ${ }_{\mathcal{B}} \mathcal{E}_{\mathcal{A}}$ is the complex conjugate bimodule ${ }_{\mathcal{A}} \bar{\varepsilon}_{\mathcal{B}}$ with its conjugate structures, see [9].

\subsection{Equivariant Picard groupoids}

In a next step we summarize some notions and results concerning equivariant (or covariant) Morita theory following [16]. In the case where the algebras under investigation possess some group or Lie algebra action, it is natural to ask for an equivariant notion of equivalence. In order to deal with groups and Lie algebras simultaneously, we adopt the more general notion of Hopf algebra actions. In the following, $H$ will denote a Hopf algebra over $\mathrm{C}$ with comultiplication $\Delta$, counit $\epsilon$,

and antipode $S$. For the comultiplication we use Sweedler's notation $\Delta(g)=g_{(1)} \otimes g_{(2)}$. Moreover, for the ${ }^{*}$-algebra case we will need in addition a ${ }^{*}$-involution for the Hopf algebra itself such that the coproduct and the counit are ${ }^{*}$-homomorphisms, i.e $\left(g_{(1)}\right)^{*} \otimes\left(g_{(2)}\right)^{*}=\left(g^{*}\right)_{(1)} \otimes\left(g^{*}\right)_{(2)}$ and $\epsilon\left(g^{*}\right)=\overline{\epsilon(g)}$. It follows that $S\left(S\left(g^{*}\right)^{*}\right)=g$ for all $g \in H$. In particular, $S$ is invertible with $S^{-1}={ }^{*} S^{*}$.

A left action of a Hopf algebra $\triangleright: H \otimes \mathcal{A} \longrightarrow \mathcal{A}$ on an algebra $\mathcal{A}$ is then a C-linear mapping with $(g h) \triangleright a=g \triangleright(h \triangleright a), h \triangleright(a b)=\left(h_{(1)} \triangleright a\right)\left(h_{(2)} \triangleright b\right), \mathbb{1}_{H} \triangleright a=a$, and $h \triangleright \mathbb{1}_{\mathcal{A}}=\epsilon(h) \mathbb{1}_{\mathcal{A}}$ for all $g, h \in H$ and $a, b \in \mathcal{A}$. In this case $\mathcal{A}$ is also called a left $H$-module algebra. If in addition $\mathcal{A}$ and $H$ possess *-structures we demand compatibility such that $(h \triangleright a)^{*}=S(h)^{*} \triangleright a^{*}$ for all $a \in \mathcal{A}$ and $h \in H$. In this case, the action will be called a left ${ }^{*}$-action. 
We now turn to $H$-equivariant representations of left $H$-module ${ }^{*}$-algebras. An $H$-equivariant bimodule ${ }_{\mathcal{B}} \mathcal{E}_{\mathcal{A}}$ is a bimodule ${ }_{\mathcal{B}} \mathcal{E}_{\mathcal{A}}$ with an $H$-action such that $h \triangleright(b \cdot x)=\left(h_{(1)} \triangleright b\right) \cdot\left(h_{(2)} \triangleright x\right)$ and $h \triangleright(x \cdot a)=\left(h_{(1)} \triangleright x\right) \cdot\left(h_{(2)} \triangleright a\right)$. The action is called compatible with given $\mathcal{A}$ - or $\mathcal{B}$-valued inner products if

$$
h \triangleright\langle x, y\rangle_{\mathcal{A}}=\left\langle S\left(h_{(1)}\right)^{*} \triangleright x, h_{(2)} \triangleright y\right\rangle_{\mathcal{A}} \quad \text { or } \quad h \triangleright{ }_{\mathcal{B}}\langle x, y\rangle={ }_{\mathcal{B}}\left\langle h_{(1)} \triangleright x, S\left(h_{(2)}\right)^{*} \triangleright y\right\rangle,
$$

respectively. If ${ }_{\mathcal{B}} \mathcal{E}_{\mathcal{A}}$ is even a (*-, strong) equivalence bimodule with compatible $H$-action, then ${ }_{\mathcal{B}} \mathcal{E}_{\mathcal{A}}$ is called a $H$-equivariant (*-, strong) equivalence bimodule and $\mathcal{A}$ and $\mathcal{B}$ are called $H$-equivariantly ${ }^{*}{ }^{*}$, strongly) Morita equivalent, respectively, see [16].

There is a canonical action on the internal tensor product of two equivariant bimodules ${ }_{e} \mathcal{F}_{\mathcal{B}}$ and ${ }_{\mathcal{B}} \mathcal{E}_{\mathcal{A}}$, namely $h \triangleright\left(x \otimes_{\mathcal{B}} y\right)=\left(h_{(1)} \triangleright x\right) \otimes_{\mathcal{B}}\left(h_{(2)} \triangleright y\right)$. If the bimodules carry compatible inner products, this action is compatible with the degeneration space of (2.1). Hence we can pass to the quotient in this case as well. In addition, given an $H$-equivariant bimodule ${ }_{\mathcal{B}} \mathcal{E}_{\mathcal{A}}$ with action $\triangleright$, there is a canonical action $\bar{\triangleright}$ on the complex conjugate ${ }_{\mathcal{A}} \bar{\varepsilon}_{\mathcal{B}}$, defined by $h \bar{\triangleright} \bar{x}=\overline{S(h)^{*} \triangleright x}$. All possible compatibilities of $\triangleright$ translate to $\triangleright$.

In all three situations (ring-theoretic, ${ }^{*}$, and strong) we obtain a well-defined composition yield-

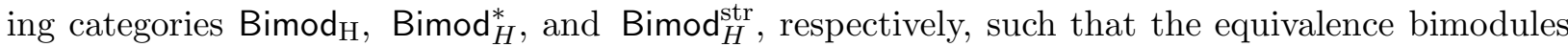
correspond to invertible arrows in these categories. This determines the corresponding equivariant Picard groupoids, see [16, Def. 4.1]:

Definition 2.1 (H-equivariant Picard groupoid) The unital $\left({ }^{*}{ }^{-}\right)$algebras over $\mathrm{C}$ with compatible (*-) action of $H$ as objects and the equivalence classes of $H$-equivariant ( ${ }^{*}$-, or strong) equivalence bimodules as morphisms form the H-equivariant (*-, or strong) Picard groupoids denoted by $\mathrm{Pic}_{H}\left(\mathrm{Pic}_{H}^{*}\right.$, or $\mathrm{Pic}_{H}^{\text {str }}$ ). The equivalence classes are constructed with $H$-equivariant (isometric) bimodule isomorphisms. The composition of the bimodules is the internal tensor product.

\subsection{The groups $\mathrm{Gl}(H, \mathcal{A})$ and $\mathrm{U}(H, \mathcal{A})$}

Forgetting the $H$-action yields natural groupoid morphism

$$
\mathrm{Pic}_{H} \longrightarrow \mathrm{Pic}, \quad \mathrm{Pic}_{H}^{*} \longrightarrow \mathrm{Pic}^{*}, \quad \text { and } \mathrm{Pic}_{H}^{\text {str }} \longrightarrow \mathrm{Pic}^{\text {str }} \text {. }
$$

We shall now recall the results from [16, Sec. 4.2] where the kernels of these groupoid morphisms were studied. The basic ingredients for the study of the kernels are the following groups as introduced in [16, Def. A.1]. As we will need them later on we give some details of the construction.

Definition 2.2 (The groups $\operatorname{Gl}(H, \mathcal{A})$ and $\mathrm{U}(H, \mathcal{A}))$ ) Let $\mathcal{A}$ be an $H$-module algebra. We call $\operatorname{Gl}(H, \mathcal{A})$ the set of all elements $\mathrm{a} \in \operatorname{Hom}_{\mathrm{C}}(H, \mathcal{A})$ such that for all $g, h \in H$ and $b \in \mathcal{A}$

i.) $\mathrm{a}\left(\mathbb{1}_{H}\right)=\mathbb{1}_{\mathcal{A}}$,

ii.) $\mathrm{a}(g h)=\mathrm{a}\left(g_{(1)}\right)\left(g_{(2)} \triangleright \mathrm{a}(h)\right)$,

iii.) $\left(h_{(1)} \triangleright b\right) \mathrm{a}\left(h_{(2)}\right)=\mathrm{a}\left(h_{(1)}\right)\left(h_{(2)} \triangleright b\right)$.

If in addition $H$ is a Hopf ${ }^{*}$-algebra, $\mathcal{A} a^{*}$-algebra and $\triangleright$ is $a^{*}$-action, we call $\mathrm{U}(H, \mathcal{A})$ the set of all those elements of $\mathrm{Gl}(H, \mathcal{A})$ such that for all $h \in H$

iv. $) \mathrm{a}\left(h_{(1)}\right)\left(\mathrm{a}\left(S\left(h_{(2)}\right)^{*}\right)\right)^{*}=\epsilon(h) \mathbb{1}_{\mathcal{A}}$. 
As has been shown in [16, Prop. A.2], these sets become groups with respect to the usual convolution product

$$
(\mathrm{a} * \mathrm{~b})(h)=\mathrm{a}\left(h_{(1)}\right) \mathrm{b}\left(h_{(2)}\right)
$$

of $\operatorname{Hom}_{\mathrm{C}}(H, \mathcal{A})$. The unit $\mathrm{e}$ is given by $\mathrm{e}(g)=\epsilon(g) \mathbb{1}_{\mathcal{A}}$. The inverse of a is given by $\mathrm{a}^{-1}(g)=$ $g_{(1)} \triangleright \mathrm{a}\left(S\left(g_{(2)}\right)\right)$. Moreover, in the ${ }^{*}$-algebra case, $\operatorname{Hom}_{\mathrm{C}}(H, \mathcal{A})$ is a unital ${ }^{*}$-algebra with respect to the convolution product and the ${ }^{*}$-involution defined by

$$
\mathrm{a}^{*}(g)=\mathrm{a}\left(S(g)^{*}\right)^{*}
$$

for $g \in H$ and $\mathrm{a} \in \operatorname{Hom}_{\mathrm{C}}(H, \mathcal{A})$. Then the last condition $i v$.) can be interpreted as unitarity in the sense that it means $\mathrm{a}^{-1}=\mathrm{a}^{*}$ for $\mathrm{a} \in \mathrm{U}(H, \mathcal{A})$.

For later use we mention the following almost trivial situation where the ${ }^{*}$-algebra $\mathcal{A}$ is commutative and the action is the trivial action.

Lemma 2.3 Let $\mathcal{A}$ be a commutative unital ${ }^{*}$-algebra (algebra) and let the ${ }^{*}$-action (action) of $H$ be trivial, i.e. $g \triangleright a=\epsilon(g) a$ for all $a \in \mathcal{A}$ and $g \in H$. Then $\mathrm{U}(H, \mathcal{A})$ consists precisely of the unital ${ }^{*}$-homomorphisms a $: H \longrightarrow \mathcal{A}$. In the ring-theoretic setting $\mathrm{Gl}(H, \mathcal{A})$ consists of the unital homomorphisms, respectively.

The relevance of these groups is now the following. Given an $H$-action $\triangleright$ on a Morita equivalence bimodule ${ }_{\mathcal{B}} \mathcal{E}_{\mathcal{A}}$, we can construct another action $\triangleright^{\mathrm{b}}$ on ${ }_{\mathcal{B}} \mathcal{E}_{\mathcal{A}}$ for all $\mathrm{b} \in \mathrm{Gl}(H, \mathcal{B})$ by

$$
h \triangleright^{\mathrm{b}} x=\mathrm{b}\left(h_{(1)}\right) \cdot\left(h_{(2)} \triangleright x\right) .
$$

Indeed, the defining properties of $b$ guarantee that this is again an action compatible with the bimodule structure. In the case of *-algebras and inner product bimodules with compatible actions, $\mathrm{b} \in \mathrm{U}(H, \mathcal{B})$ yields an action again compatible with the inner products. On the other hand, if two $H$-actions $\triangleright$ and $\triangleright^{\prime}$ are given,

$$
u_{h}(x)=h_{(1)} \triangleright\left(S\left(h_{(2)}\right) \triangleright^{\prime} x\right)
$$

for $h \in H$ and $x \in{ }_{\mathcal{B}} \mathcal{E}_{\mathcal{A}}$ defines a right $\mathcal{A}$-linear endomorphism $u_{h}$ of ${ }_{\mathcal{B}} \mathcal{E}_{\mathcal{A}}$. Because we work in the unital case, $u_{h}$ is the left multiplication by a unique element of $\mathcal{B}$, which we denote by $\mathrm{b}(h)$. It turns out that $\mathrm{b} \in \mathrm{Gl}(H, \mathcal{B})$ or, for *-equivalence bimodules, $\mathrm{b} \in \mathrm{U}(H, \mathcal{B})$ if the actions were compatible with the inner products. It is now easy to see that this gives indeed a bijection and hence the elements of $\mathrm{Gl}(H, \mathcal{B})$ (or $\mathrm{U}(H, \mathcal{B})$ ) parametrize the possible compatible $H$-actions on a given equivalence bimodule (or *-equivalence bimodule, respectively), provided there is at least one compatible action at all. In fact, the above construction gives a free and transitive group action of $\mathrm{Gl}(H, \mathcal{B})$ (or $\mathrm{U}(H, \mathcal{B}))$ on the set of possible compatible $H$-actions.

Though $\mathrm{Gl}(H, \mathcal{B})$ (or $\mathrm{U}(H, \mathcal{B})$, respectively) acts freely and transitively on the set of all compatible $H$-actions, some of them might lead to isomorphic bimodules and hence to the same element in the Picard groupoid. They can be described as follows: We denote by $G L(\mathfrak{Z}(\mathcal{B}))$ the group of all central and invertible elements of $\mathcal{B}$ and by $U(\mathfrak{Z}(\mathcal{B}))$ the group of all central and unitary elements, respectively. The subgroups of $H$-invariant elements are denoted by $\operatorname{GL}(\mathfrak{Z}(\mathcal{B}))^{H}$ and $\mathbf{U}(\mathfrak{Z}(\mathcal{B}))^{H}$, respectively. There is a group morphism

$$
\mathrm{GL}(\mathfrak{Z}(\mathcal{B})) \ni c \longmapsto\left(\hat{c}: h \longmapsto c\left(h \triangleright c^{-1}\right)\right) \in \mathrm{Gl}(H, \mathcal{B}) .
$$

Then $\triangleright$ and $\triangleright^{\mathrm{b}}$ define isomorphic $H$-equivariant equivalence bimodules iff $\mathrm{b}$ is in the image of (2.8). The analogous result holds for the case of ${ }^{*}$-algebras and the groups $\mathrm{U}(\mathfrak{Z}(\mathcal{B}))$ and $\mathbf{U}(H, \mathcal{B})$ instead of $\mathrm{GL}(\mathfrak{Z}(\mathcal{B}))$ and $\mathrm{Gl}(H, \mathcal{B})$. It follows that the image of (2.8) is a normal subgroup such that the 
quotient groups $\left.\mathrm{Gl}_{0}(H, \mathcal{B})=\mathrm{Gl}(H, \mathcal{B}) / \mathrm{GL} \widehat{(\mathfrak{Z}(\mathcal{B})}\right)$ and $\left.\mathrm{U}_{0}(H, \mathcal{B})=\mathrm{U}(H, \mathcal{B}) / \widehat{\mathrm{U}(\widehat{\mathfrak{Z}(\mathcal{B}})}\right)$ parametrize the inequivalent actions on an equivalence bimodule. In particular, we obtain short exact sequences

$$
1 \longrightarrow \mathrm{Gl}_{0}(H, \mathcal{B}) \longrightarrow \operatorname{Pic}_{H}(\mathcal{B}) \longrightarrow \operatorname{Pic}(\mathcal{B})
$$

in the ring-theoretic situation and

$$
1 \longrightarrow \mathrm{U}_{0}(H, \mathcal{B}) \longrightarrow \mathrm{Pic}_{H}^{*}(\mathcal{B}) \longrightarrow \operatorname{Pic}^{*}(\mathcal{B})
$$

in the ${ }^{*}$-algebra framework, thereby encoding the kernels of the groupoid morphisms $\mathrm{Pic}_{H} \longrightarrow$ Pic and $\mathrm{Pic}_{H}^{*} \longrightarrow \mathrm{Pic}^{*}$. Since the complete positivity of inner products was not playing any role in the above argument, the analogous statement also holds for the strong version.

Similarly, there is an action of $\mathrm{a} \in \mathrm{GI}(H, \mathcal{A})$ (or a $\in \mathrm{U}(H, \mathcal{A})$, respectively) from the right on $\left({ }_{B} \mathcal{E}_{\mathcal{A}}, \triangleright\right)$ by twisting the $H$-action $\triangleright$ to $g \triangleright_{\mathrm{a}} x=\left(g_{(1)} \triangleright x\right) \cdot \mathrm{a}\left(g_{(2)}\right)$ for $g \in H$ and $x \in{ }_{\mathcal{B}} \mathcal{E}_{\mathcal{A}}$. This can be used to see that the groups $\mathrm{Gl}(H, \mathcal{B})$ and $\mathrm{Gl}(H, \mathcal{A})$ are actually isomorphic for $H$-equivariantly Morita equivalent algebras. Analogously $\mathrm{U}(H, \mathcal{B})$ and $\mathrm{U}(H, \mathcal{A})$ are isomorphic for $H$-equivariantly *-Morita equivalent ${ }^{*}$-algebra. For later use we recall the relation between the two groups for the *-algebra case:

Lemma 2.4 Let ${ }_{\mathcal{B}} \mathcal{E}_{\mathcal{A}} \in \operatorname{Pic}_{H}^{*}(\mathcal{B}, \mathcal{A})$ and $\mathrm{b} \in \mathrm{U}(H, \mathcal{B})$ and $\mathrm{a} \in \mathrm{U}(H, \mathcal{A})$. We have $\left(\triangleright_{\mathrm{a}}\right)^{\mathrm{b}}=\left(\triangleright^{\mathrm{b}}\right)_{\mathrm{a}}$ and there exists a unique $h_{\mathcal{E}}(\mathrm{a}) \in \mathrm{U}(H, \mathcal{B})$, such that $\triangleright_{\mathrm{a}}=\triangleright^{h_{\mathcal{E}}(\mathrm{a})}$. The map

$$
h_{\mathcal{E}}: \mathrm{U}(H, \mathcal{A}) \ni \mathrm{a} \longmapsto h_{\mathcal{E}}(\mathrm{a}) \in \mathrm{U}(H, \mathcal{B})
$$

is a group isomorphism and determines a group isomorphism $\mathrm{U}_{0}(H, \mathcal{A}) \longrightarrow \mathrm{U}_{0}(H, \mathcal{B})$ that will also be denoted by $h_{\mathcal{E}}$.

In fact, $h_{\varepsilon}$ turns out to come from a groupoid action of $\mathrm{Pic}_{H}^{*}$ on the collection of all the groups $\mathrm{U}(H, \cdot)$ in a very precise sense, see [16, Lem. 5.11, Thm 5.14] for details and the proof of this lemma.

\section{$3 \quad$ Fedosov star products and quantum momentum maps}

While the above considerations apply to general algebras and *-algebras, respectively, we focus now on the star product algebras arising from deformation quantization [3]. To keep things simple, we consider star products on a symplectic manifold which we assume to be connected. We recall the basic notions.

\subsection{Deformation quantization}

A star product $\star$ is a $\mathbb{C}[[\lambda]]$-bilinear associative multiplication for the formal power series with coefficients in the complex-valued functions $C^{\infty}(M)[[\lambda]]$ written as

$$
f \star g=\sum_{r=0}^{\infty} \lambda^{r} C_{r}(f, g)
$$

for $f, g \in C^{\infty}(M)[[\lambda]]$ such that $C_{0}(f, g)=f g$ is the pointwise (commutative) product and $C_{1}(f, g)-C_{1}(g, f)=\mathrm{i}\{f, g\}$ yields the Poisson bracket. Moreover, we require $\star$ to be differential, i.e. all the maps $C_{r}$ should be bidifferential operators on $M$. Finally, one requires $1 \star f=f=f \star 1$. A star product is called Hermitian if in addition $\overline{f \star g}=\bar{g} \star \bar{f}$. Here the complex conjugation of $\lambda$ is defined to be $\bar{\lambda}=\lambda$. Thus a Hermitian star product turns $C^{\infty}(M)[[\lambda]]$ into a unital *algebra 
over $\mathbb{C}[[\lambda]]$ and we arrive at the general framework discussed before. Finally, recall that two star products $\star$ and $\star^{\prime}$ are called equivalent if there is a formal series $T=\mathrm{id}+\sum_{r=1}^{\infty} \lambda^{r} T_{r}$ of differential operators such that $f \star^{\prime} g=T^{-1}(T f \star T g)$, i.e. $T$ is an algebra isomorphism deforming the identity. On a symplectic manifold, there is a characteristic class which assigns to every $\star$ a formal series $c(\star) \in \frac{[\omega]}{\mathrm{i} \lambda}+\mathrm{H}_{\mathrm{dR}}^{2}(M, \mathbb{C})[[\lambda]]$ in the second deRham cohomology such that two star products are equivalent iff their characteristic classes coincide, see e.g. [13] for a nice introduction. Every such formal series arises as the characteristic class of a star product. Note that the characteristic class is natural with respect to symplectomorphisms, i.e. $\Psi^{*} c(\star)=c\left(\Psi^{*}(\star)\right)$, when we move around the star product by means of $\Psi$. A pedagogical introduction to deformation quantization and further references can be found in [23].

In the following we shall make extensive use of the Fedosov construction [11] of star products on a symplectic manifold. Fedosov constructed out of a symplectic torsion-free connection $\nabla$ and a formal series of closed two forms $\Omega \in \lambda \Omega^{2}(M)[[\lambda]]$ a differential star product, the Fedosov star product $\star_{\nabla, \Omega}$. It is Hermitian iff $\bar{\Omega}=\Omega$ is real. It turns out that $c\left(\star_{\nabla, \Omega}\right)=\frac{1}{\mathrm{i} \lambda}[\omega+\Omega]$, see e.g. [23, Sect. 6.4] for further details. We shall also need the following simple generalization of Fedosov's construction [21]: let $E \longrightarrow M$ be a complex vector bundle over a symplectic manifold $M$ and choose a covariant derivative $\nabla^{E}$ for it. Then one can construct a formal series of bidifferential operators $R_{r}: \Gamma^{\infty}(E) \times C^{\infty}(M) \longrightarrow \Gamma^{\infty}(E)$ such that

$$
s \bullet f=s \cdot f+\sum_{r=1}^{\infty} \lambda^{r} R_{r}(s, f)
$$

for $s \in \Gamma^{\infty}(E)[[\lambda]]$ and $f \in C^{\infty}(M)[[\lambda]]$ defines a deformation of the classical right module structure into a right module structure with respect to $\star \nabla, \Omega$. Moreover, there exists a formal series of bidifferential operators $C_{r}^{\prime}: \Gamma^{\infty}(\operatorname{End}(E)) \times \Gamma^{\infty}(\operatorname{End}(E)) \longrightarrow \Gamma^{\infty}(\operatorname{End}(E))$ such that

$$
A \star^{\prime} B=A B+\sum_{r=1}^{\infty} \lambda^{r} C_{r}^{\prime}(A, B)
$$

for $A, B \in \Gamma^{\infty}(\operatorname{End}(E))[[\lambda]]$ defines a formal associative deformation. Finally, there is also a bidifferential deformed left module structure

$$
A \bullet^{\prime} s=A s+\sum_{r=1}^{\infty} \lambda^{r} R_{r}^{\prime}(A, s)
$$

for $A \in \Gamma^{\infty}(\operatorname{End}(E))[[\lambda]]$ and $s \in \Gamma^{\infty}(E)[[\lambda]]$ with respect to $\star^{\prime}$ such that in total we obtain a deformed bimodule structure. In fact, this turns out to be a ring-theoretic equivalence bimodule and all equivalence bimodules with $C^{\infty}(M)[[\lambda]]$ acting from the right are isomorphic to such a deformed vector bundle. For the classical limit this is of course well-known. In the case $E=L$ is a line bundle, $\star^{\prime}$ yields a star product, too, and the deformed line bundle is an equivalence bimodule for $\star$ and $\star^{\prime}$.

In addition, one can specify a pseudo Hermitian fiber metric $h$ on $E$, viewed as an inner product $h: \Gamma^{\infty}(E) \times \Gamma^{\infty}(E) \longrightarrow C^{\infty}(M)$. This endows $\Gamma^{\infty}(\operatorname{End}(E))$ with a *involution. Next, if we assume that $\nabla^{E}$ is compatible with $h$, the Fedosov construction automatically yields a *equivalence bimodule by suitably deforming $h$ as well. If in addition $h$ was a Hermitian fiber metric then the deformed *-equivalence bimodule is even a strong one. Again, all are of this form up to isomorphisms. 


\subsection{Quantum momentum maps}

We will now start to incorporate symmetries into this situation. In the following we will always assume that there is a Lie algebra $\mathfrak{g}$ acting on $(M, \omega)$ by symplectic vector fields denoted by $X_{\xi}$ where $\xi \in \mathfrak{g}$. We follow the convention that $\rho(\xi)=-\mathcal{L}_{X_{\xi}}$ defines a left representation of $\mathfrak{g}$ on $C^{\infty}(M)$, where $\mathcal{L}$ denotes the usual Lie derivative. Thus $\rho(\xi)$ is a Poisson derivation. The typical situation we have in mind is that the $X_{\xi}$ are the fundamental vector fields of a symplectic Lie group action. However, in the following we never need the integrated version but exclusively use the Lie algebraic point of view. This opens the framework also to more general Lie algebra actions where the fundamental vector fields may be non-complete. However, throughout this section, we do assume that on $M$ there is a $\mathfrak{g}$-invariant connection. In this case, there is also a $\mathfrak{g}$-invariant symplectic torsion-free connection $\nabla$.

To cast this situation into the general Hopf *-algebra framework we consider the following two Hopf algebras: first, we take the formal power series in the complexified tensor algebra over $\mathfrak{g}$, viewed as a $\mathbb{C}[[\lambda]]$-module, and divide by the two-sided ideal generated by the relations $\xi \otimes \eta-\eta \otimes \xi-\mathrm{i} \lambda[\xi, \eta]$ for all $\xi, \eta \in \mathfrak{g}$. The resulting "rescaled" universal enveloping algebra will be denoted by $\mathcal{U}_{\lambda}(\mathfrak{g})$. The $\mathrm{i}$ in front of the bracket allows to consider the elements $\xi \in \mathfrak{g} \subseteq \mathcal{U}_{\lambda}(\mathfrak{g})$ to be Hermitian, the reason for the additional $\lambda$ will become clear in (3.11). The requirements

$$
\xi^{*}=\xi, \quad \Delta(\xi)=\xi \otimes \mathbb{1}+\mathbb{1} \otimes \xi, \quad \epsilon(\xi)=0, \quad \text { and } \quad S(\xi)=-\xi
$$

for all $\xi \in \mathfrak{g}$ lead to a unique Hopf *-algebra structure for $\mathcal{U}_{\lambda}(\mathfrak{g})$ over $\mathbb{C}[[\lambda]]$. Second, the complexified universal enveloping algebra $\mathcal{U}_{\mathbb{C}}(\mathfrak{g})$ is a Hopf ${ }^{*}$-algebra, too, with ${ }^{*}$-involution determined by $\xi^{*}=-\xi$. To be in the framework of Hopf ${ }^{*}$-algebras over $\mathbb{C}[[\lambda]]$ we extend all structure maps to $\mathcal{U}_{\mathbb{C}}(\mathfrak{g})[[\lambda]]$

We have to strengthen now the assumptions about the action in the following way: Recall that, on the classical level, the Lie algebra action is called Hamiltonian if there is a linear mapping $J_{0}: \mathfrak{g} \longrightarrow C^{\infty}(M)$ with $\mathrm{i}_{X_{\xi}} \omega=\mathrm{d} J_{0}(\xi)$. Equivalently, this means that the Poisson derivations $\rho(\xi)=\left\{J_{0}(\xi), \cdot\right\}$ are inner. If in addition $J_{0}$ is equivariant with respect to the adjoint action of $\mathfrak{g}$, which is equivalent to

$$
J_{0}([\xi, \eta])=\left\{J_{0}(\xi), J_{0}(\eta)\right\} \quad \text { for all } \quad \xi, \eta \in \mathfrak{g},
$$

then $J_{0}$ is called classical momentum map.

The appropriate notion of symmetry in deformation quantization is now the following, see e.g. 4] and references therein. A star product $\star$ is called $\mathfrak{g}$-invariant if the action $\rho$ of $\mathfrak{g}$ is given by derivations of $\star$. The Fedosov construction depends functorially on the data $\nabla, \Omega$. This allows to show that $\star_{\nabla, \Omega}$ is $\mathfrak{g}$-invariant iff the entrance data $\nabla$ and $\Omega$ are $\mathfrak{g}$-invariant. Moreover, one can show that every $\mathfrak{g}$-invariant star product $\star$ is $\mathfrak{g}$-equivariantly equivalent to a $\mathfrak{g}$-invariant Fedosov star product $\star \nabla, \Omega$. Finally, one knows that $\star \nabla, \Omega$ is $\mathfrak{g}$-equivariantly equivalent to $\star_{\nabla^{\prime}, \Omega^{\prime}}$ iff $\Omega$ and $\Omega^{\prime}$ are cohomologous in the $\mathfrak{g}$-invariant deRham cohomology, see [4 for detailed proofs for the case of a Lie group action: the Lie algebraic case can be done by completely analogous means. This allows to define the $\mathfrak{g}$-invariant characteristic class

$$
c^{\mathfrak{g}}(\star)=\frac{[\omega+\Omega]}{\mathrm{i} \lambda} \in \frac{[\omega]}{\mathrm{i} \lambda}+\mathrm{H}_{\mathrm{dR}}^{2}(M, \mathbb{C})^{\mathfrak{g}}[[\lambda]],
$$

where $\Omega$ is a formal series of $\mathfrak{g}$-invariant closed two-forms such that $\star$ is $\mathfrak{g}$-equivariantly equivalent to $\star \nabla, \Omega$. Note that under the canonical map

$$
\mathrm{H}_{\mathrm{dR}}^{2}(M, \mathbb{C})^{\mathfrak{g}} \longrightarrow \mathrm{H}_{\mathrm{dR}}^{2}(M, \mathbb{C}),
$$


extended to (3.7), the $\mathfrak{g}$-invariant characteristic class $c^{\mathfrak{g}}(\star)$ is mapped to the characteristic class $c(\star)$. In particular, an arbitrary star product $\star$ is equivalent to a $\mathfrak{g}$-invariant star product iff $c(\star)$ is in the image of (3.8). Note also that the construction of $c^{\mathfrak{g}}(\cdot)$ requires the existence of a $\mathfrak{g}$-invariant connection but does not depend on the particular choice of it.

In the following, we assume that a classical momentum map $J_{0}$ exists and is given. Then the analog of a classical momentum map is a quantum momentum map, see [20] and references therein:

Definition 3.1 (Quantum momentum map) Let $\star$ be a $\mathfrak{g}$-invariant star product. A linear map $J=J_{0}+J_{+}: \mathfrak{g} \longrightarrow C^{\infty}(M)[[\lambda]]$ with $J_{0}: \mathfrak{g} \longrightarrow C^{\infty}(M)$ and $J_{+}: \mathfrak{g} \longrightarrow \lambda C^{\infty}(M)[[\lambda]]$ is called quantum Hamiltonian for the action $\rho$ if

$$
\rho(\xi)=\frac{1}{\mathrm{i} \lambda} \operatorname{ad}_{\star}(J(\xi)) \quad \text { for all } \quad \xi \in \mathfrak{g} .
$$

Moreover, $J$ is called a quantum momentum map if in addition for all $\xi, \eta \in \mathfrak{g}$

$$
\mathrm{i} \lambda J([\xi, \eta])=[J(\xi), J(\eta)]_{\star} .
$$

In the case where $\star$ is Hermitian we require in addition $J(\xi)^{*}=J(\xi)$.

Note that the right hand side of (3.10) is necessarily of order $\lambda$ making the condition meaningful. Thanks to this and thanks to the definition of the rescaled universal enveloping algebra, a quantum momentum map uniquely extends to a unital $\mathbb{C}[[\lambda]]$-linear algebra homomorphism

$$
J: \mathcal{U}_{\lambda}(\mathfrak{g}) \longrightarrow C^{\infty}(M)[[\lambda]]
$$

which is even a ${ }^{*}$-homomorphism as soon as $\star$ is Hermitian. Moreover, the definition

$$
\xi \triangleright f=\mathrm{i} \lambda \rho(\xi) f
$$

for $\xi \in \mathfrak{g}$ and $f \in C^{\infty}(M)[[\lambda]]$ extends to a Hopf algebra action (even a *action in the Hermitian case) of $\mathcal{U}_{\lambda}(\mathfrak{g})$ on $C^{\infty}(M)[[\lambda]]$ as soon as $\star$ is $\mathfrak{g}$-invariant.

Since we assume to have a $\mathfrak{g}$-invariant connection, one even has necessary and sufficient conditions for the existence of quantum momentum maps for $\mathfrak{g}$-invariant Fedosov star products. In fact, there exists a quantum momentum map for the $\mathfrak{g}$-invariant Fedosov star product $\star_{\nabla, \Omega}$ if and only if there exists a cochain $J \in C^{1}\left(\mathfrak{g}, C^{\infty}(M)\right)[[\lambda]]$ such that

$$
\mathrm{d} J(\xi)=\mathrm{i}_{X_{\xi}}(\omega+\Omega) \quad \text { and } \quad(\omega+\Omega)\left(X_{\xi}, X_{\eta}\right)=J([\xi, \eta]) \quad \text { for all } \xi, \eta \in \mathfrak{g} .
$$

Moreover, in this case the quantum momentum map $J$ is determined up to Chevalley cocycles in $Z^{1}(\mathfrak{g}, \mathbb{C})[[\lambda]]$. In case of a Hermitian Fedosov star product, i.e. $\bar{\Omega}=\Omega$, the real quantum momentum map is determined up to Chevalley cocycles in $Z^{1}(\mathfrak{g}, \mathbb{R})[[\lambda]]$, see [20] for detailed proofs of these statements.

For any other $\mathfrak{g}$-invariant star product $\star$ we can pass to the corresponding Fedosov star product $\star \nabla, \Omega$ by means of a $\mathfrak{g}$-equivariant equivalence transformation thanks to the above classification, see [4, Prop. 4.1]. So if

$$
f \star \nabla, \Omega g=T\left(T^{-1}(f) \star T^{-1}(g)\right)
$$

denotes such a $\mathfrak{g}$-equivariant equivalence transformation $T$, it is easy to see that $J$ is a quantum momentum map for $\star$ if and only if $T J$ is a quantum momentum map for $\star \nabla, \Omega$. In fact, one can transport quantum momentum maps with equivariant equivalence transformations to any other equivalent star product as well. Thus the existence of a quantum momentum map is a property of the $\mathfrak{g}$-invariant characteristic class $c^{\mathfrak{g}}(\star)$ within the $\mathfrak{g}$-invariant star products and not just of the star product itself. 


\subsection{Equivariant cohomology}

We will now rephrase the above well-known conditions for the existence of quantum momentum maps in terms of equivariant cohomology. To this end, recall the Cartan model for equivariant cohomology, see e.g. [12 for details.

The $\mathfrak{g}$-equivariant differential forms of degree $k$ are elements of the space

$$
\Omega_{\mathfrak{g}}^{k}(M, \mathbb{C})=\bigoplus_{2 i+j=k}\left(\mathrm{~S}^{i}\left(\mathfrak{g}^{*}\right) \otimes \Omega^{j}(M, \mathbb{C})\right)^{\mathfrak{g}}
$$

Here $S^{i}\left(\mathfrak{g}^{*}\right)$ denotes the (complexified) symmetric tensors over $\mathfrak{g}^{*}$ endowed with the inherited coadjoint action of $\mathfrak{g}$. We usually view them as homogeneous polynomial maps from $\mathfrak{g}$ to $\mathbb{C}$ of degree $i$. Thus we can view $\alpha \in \Omega_{G}^{k}(M, \mathbb{C})$ as a polynomial subject to the equivariance condition

$$
\alpha([\xi, \eta])=\mathcal{L}_{X_{\xi}} \alpha(\eta) \text { for all } \xi, \eta \in \mathfrak{g} .
$$

The differential $\mathrm{d}_{\mathfrak{g}}: \Omega_{\mathfrak{g}}^{k}(M, \mathbb{C}) \longrightarrow \Omega_{\mathfrak{g}}^{k+1}(M, \mathbb{C})$ is defined to be

$$
\left(\mathrm{d}_{\mathfrak{g}} \alpha\right)(\xi)=\mathrm{d}(\alpha(\xi))+\mathrm{i}\left(X_{\xi}\right) \alpha(\xi) .
$$

Then the Cartan model of equivariant cohomology is defined to be the cohomology of this complex, i.e.

$$
\mathrm{H}_{\mathfrak{g}}^{\bullet}(M, \mathbb{C})=\operatorname{ker} \mathrm{d}_{\mathfrak{g}} / \operatorname{im~d}_{\mathfrak{g}},
$$

which is graded as $\mathrm{d}_{\mathfrak{g}}$ is compatible with the degree $k$. In the following we will exclusively work with the Cartan model of equivariant cohomology and simply call this the equivariant cohomology of $M$ with respect to the Lie algebra action of $\mathfrak{g}$. Note that $\mathrm{d}_{\mathfrak{g}}$ commutes with complex conjugation and hence allows for a real equivariant cohomology as well.

Remark 3.2 Note that we do not require that the Lie algebra action integrates to a Lie group action, contrary to [12. Even if it integrates, the corresponding Lie group action is allowed to be quite arbitrary. In particular, we do not need it to be proper. As already mentioned, for our purposes it will turn out that the existence of the invariant connection is all we need which is of course a strictly weaker requirement than a proper action.

We are mainly interested in the second equivariant cohomology. In degree $k=1$ and $k=2$, the equivariant forms are

$$
\Omega_{\mathfrak{g}}^{1}(M, \mathbb{C})=\Omega^{1}(M, \mathbb{C})^{\mathfrak{g}} \quad \text { and } \quad \Omega_{\mathfrak{g}}^{2}(M, \mathbb{C})=\Omega^{2}(M, \mathbb{C})^{\mathfrak{g}} \oplus C^{1}\left(\mathfrak{g}, C^{\infty}(M)\right)^{\mathfrak{g}}
$$

From this we see that projecting to the first component in a closed equivariant two-form yields a well-defined canonical map

$$
\mathrm{H}_{\mathfrak{g}}^{2}(M, \mathbb{C}) \longrightarrow \mathrm{H}_{\mathrm{dR}}^{2}(M, \mathbb{C})^{\mathfrak{g}} .
$$

Furthermore, a closed two-form $\Omega \in \Omega^{2}(M)$ can be extended to a closed equivariant two-form if and only if there is a $J \in C^{1}\left(\mathfrak{g}, C^{\infty}(M)\right)$ such that

$$
\mathcal{L}_{X_{\xi}} \Omega=0, \quad \mathrm{i}_{X_{\xi}} \Omega=\mathrm{d} J(\xi), \quad \text { and } \quad J([\xi, \eta])=\Omega\left(X_{\xi}, X_{\eta}\right)
$$

for all $\xi, \eta \in \mathfrak{g}$. Using this, we can reformulate the existence of quantum momentum maps in terms of the equivariant cohomology. 
Proposition 3.3 Let $\mathfrak{g}$ act on $(M, \omega)$ symplectically with classical momentum map $J_{0}$. Assume furthermore that there exists a $\mathfrak{g}$-invariant symplectic connection $\nabla$ for $M$. Then for a $\mathfrak{g}$-invariant star product $\star$ there exists a quantum momentum map if and only if $c^{\mathfrak{g}}(\star)$ is in the image of (3.20).

Proof. First assume $\star$ is equal to the Fedosov star product for $\Omega$ and $\nabla$. Then the assertion follows from the characterization (3.13), see [20]. Since every $\mathfrak{g}$-invariant star product is $\mathfrak{g}$-invariantly equivalent to a Fedosov star product and since $c^{\mathfrak{g}}(\star)$ is precisely defined by means of the corresponding Fedosov data, the general case follows as well by transporting the quantum momentum map via the equivalence.

The corresponding characterization of the existence of a classical momentum map is well-known, see e.g. the discussion in [12, Chap. 9].

Example 3.4 (The Weyl-Moyal star product) Let us discuss an illustrative and well-known example for invariant star products. For convenience, we formulate this example also in the global way using Lie group actions. We consider $\mathbb{R}^{2 n}$ with the standard symplectic form $\omega$ and the canonical symplectic action of the affine symplectic group $\operatorname{Sp}_{2 n} \ltimes \mathbb{R}^{2 n}$. Note that this group action is not proper, the canonical flat connection is, however, invariant with respect to this group action. The invariant second deRham cohomology $\mathrm{H}_{\mathrm{dR}}^{2}\left(\mathbb{R}^{2 n}, \mathbb{C}\right)^{\mathrm{Sp}_{2 n} \ltimes \mathbb{R}^{2 n}} \cong \mathbb{C}[\omega]$ is one dimensional. In particular, (3.8) is not injective in this case (as it would be for compact groups). The map $J_{\mathrm{Sp}}(\xi)(x)=-\frac{1}{2} x^{T} \omega \xi x$ for $\xi$ in the Lie algebra $\mathfrak{s p}_{2 n}$ of $\mathrm{Sp}_{2 n}$ and $x \in \mathbb{R}^{2 n}$ defines a classical momentum map for the action of $\mathrm{Sp}_{2 n}$. In contrast, the infinitesimal action of the translations is Hamiltonian with respect to the map $J_{\mathbb{R}^{2 n}}(a)(x)=-x^{T} \omega a$ for $a, x \in \mathbb{R}^{2 n}$ but with absence of $\mathrm{ad}^{*}$-equivariance. The Weyl-Moyal star product

$$
f \star_{0} g=\sum_{r=0}^{\infty} \frac{1}{r !}\left(-\frac{\mathrm{i} \lambda}{2}\right)^{r} \omega^{i_{1} j_{1}} \cdots \omega^{i_{r} j_{r}} \frac{\partial^{r} f}{\partial x^{i_{1}} \cdots \partial x^{i_{r}}} \frac{\partial^{r} g}{\partial x^{j_{1}} \cdots \partial x^{j_{r}}},
$$

with $\omega^{i j}$ being the inverse matrix of the coefficient matrix of $\omega$, is invariant with respect to the action of $\mathrm{Sp}_{2 n} \ltimes \mathbb{R}^{2 n}$. Moreover, $J_{\mathrm{Sp}}(\xi)$ is a quantum momentum map for the $\mathrm{Sp}_{2 n}$ action, whereas $J_{\mathbb{R}^{2 n}}$ yields only a quantum Hamiltonian for the translations. By $\star_{c \omega}$ for $c \in \lambda \mathbb{C}[[\lambda]]$ we denote the "WeylMoyal" star products for the rescaled symplectic form $(1+c) \omega$, i.e. the Fedosov star products to the choice $\Omega=c \omega$. It can be shown that these are the only $\mathrm{Sp}_{2 n} \ltimes \mathbb{R}^{2 n}$-invariant star products on $\mathbb{R}^{2 n}$ : this follows from the fact that any such invariant star product has to be equivalent to a Fedosov one and the fact that there are simply no candidates for invariant equivalence transformations beside the identity due to the lack of invariant differential operators. Clearly $(1+c) J_{\mathrm{Sp}}$ yields a quantum momentum map for $\star_{c \omega}$ for the $\mathrm{Sp}_{2 n}$-action. In particular, the Weyl-Moyal star product $\star_{0}$ is the unique $\mathrm{Sp}_{2 n} \ltimes \mathbb{R}^{2 n}$-invariant star product with the classical momentum map for the $\mathrm{Sp}_{2 n}$-action as quantum momentum map. This feature is sometimes referred to as strong invariance. The formal exponential of the Euler vector field leads to $\mathrm{Sp}_{2 n}$-invariant equivalences between the different star products $\star_{c \omega}$, as expected from the equation $[\omega]=[0]$ in $\mathrm{H}_{\mathrm{dR}}^{2}\left(\mathbb{R}^{2 n}\right)^{\mathrm{Sp}_{2 n}}$.

\section{Momentum maps and the splitting}

In this section we will generalize the notion of a (quantum) momentum map to the general algebraic situation of Hopf algebra actions. This will allow us to prove a general splitting theorem for the groupoid morphisms (2.3). 


\subsection{Momentum maps}

The image of (2.3) is, in general, very hard to understand and corresponds to a "lifting" problem: the $H$-action has to be lifted from the algebras to the bimodule, see the discussion in [16, Ex. 4.8]. However, if we restrict to a subclass of algebras having a momentum map things turn out to be very nice. The following axiomatization of a (quantum) momentum map is now straightforward:

Definition 4.1 (Momentum map) Let $\mathcal{A}$ be a unital ${ }^{*}$-algebra with $a^{*}$-action of a Hopf ${ }^{*}$ algebra $H$. Then a momentum map $J$ is a ${ }^{*}$-homomorphism $J: H \longrightarrow \mathcal{A}$ such that for all $h \in H$ and $a \in \mathcal{A}$

$$
h \triangleright a=J\left(h_{(1)}\right) a J\left(S\left(h_{(2)}\right)\right) .
$$

The set of all such momentum maps for the given ${ }^{*}$-action is denoted by $\operatorname{MoMa}_{H}^{*}(\mathcal{A})$.

Conversely, if a *-homomorphism $J: H \longrightarrow \mathcal{A}$ is given, then (4.1) defines a *-action of $H$ on $\mathcal{A}$. In the ring-theoretic case we drop the requirement $J\left(g^{*}\right)=J(g)^{*}$ and obtain the analogous definition for a momentum map. Then the set of all momentum maps is denoted by $\operatorname{MoMa}_{H}(\mathcal{A})$, respectively.

Note that the action of $H$ is inner, but the choice of the inner elements is even consistent with the algebra structure of $H$. This corresponds to the equivariance requirement of classical momentum maps in symplectic geometry. Note also that a momentum map for a non-trivial action of $H$ requires $\mathcal{A}$ to be sufficiently noncommutative: if the action is inner, the center of $\mathcal{A}$ consists of $H$-invariant elements, i.e.

$$
\mathfrak{Z}(\mathcal{A}) \subseteq \mathcal{A}^{H} .
$$

There is no a priori restriction on the Hopf algebra, as the adjoint action of any Hopf algebra on itself is just an inner action with the identity as momentum map.

For a star product algebra $\left(C^{\infty}(M)[[\lambda]], \star\right)$, this notion of a momentum map for $H=\mathcal{U}_{\lambda}(\mathfrak{g})$ coincides with the notion of a quantum momentum map if the action is of the form (3.12). Note that it is crucial to use the rescaled universal enveloping algebra since for a star product algebra (4.1) necessarily vanishes in zeroth order according to the commutativity of the undeformed algebra.

In the case when we have a specific momentum map we can simplify the groups $\mathrm{U}(H, \mathcal{A})$ and $\mathrm{GI}(H, \mathcal{A})$ further. In fact, only central elements are needed. To see this, we proceed in several steps:

Lemma 4.2 Let $\mathcal{A}$ be a unital ${ }^{*}$-algebra with $a^{*}$-action of $H$ and a momentum map $J$.

i.) A central element $z \in \mathfrak{Z}(\mathcal{A})$ is invariant under the action, i.e. $g \triangleright z=\epsilon(g) z$ for all $g \in H$.

ii.) The group $\mathrm{U}(H, \mathfrak{Z}(\mathcal{A}))$ coincides with the unital ${ }^{*}$-homomorphisms $\mathrm{z}: H \longrightarrow \mathfrak{Z}(\mathcal{A})$.

iii.) The map

$$
\mathrm{U}(H, \mathcal{A}) \ni \mathrm{a} \longmapsto \mathrm{a}_{J}=J^{-1} * \mathrm{a} * J \in \mathrm{U}(H, \mathfrak{Z}(\mathcal{A}))
$$

is an isomorphism of groups. Here $J^{-1}(g)=J(S(g))$ is the convolution inverse of $J$.

In the ring-theoretic situation the analogous statement holds with $\mathrm{U}(H, \mathcal{A})$ and $\mathrm{U}(H, \mathfrak{Z}(\mathcal{A})$ ) being replaced by $\mathrm{Gl}(H, \mathcal{A})$ and $\mathrm{Gl}(H, \mathfrak{Z}(\mathcal{A}))$, respectively.

Proof. The first part was already noted in (4.2). Since $\mathfrak{Z}(\mathcal{A})$ is commutative by definition, we can apply Lemma 2.3 to obtain the second part. For the last part, we show that $\mathrm{a}_{J}(g)$ is central for 
every $g \in H$. Indeed, for all $b \in \mathcal{A}$ we have

$$
\begin{aligned}
\mathrm{a}_{J}(g) b & =J\left(S\left(g_{(1)}\right)\right) \mathrm{a}\left(g_{(2)}\right) J\left(g_{(3)}\right) b \\
& =J\left(S\left(g_{(1)}\right)\right) \mathrm{a}\left(g_{(2)}\right)\left(g_{(3)} \triangleright b\right) J\left(g_{(4)}\right) \\
& =J\left(S\left(g_{(1)}\right)\right)\left(g_{(2)} \triangleright b\right) \mathrm{a}\left(g_{(3)}\right) J\left(g_{(4)}\right) \\
& =b J\left(S\left(g_{(1)}\right)\right) \mathrm{a}\left(g_{(2)}\right) J\left(g_{(3)}\right) \\
& =b \mathrm{a}_{J}(g),
\end{aligned}
$$

using the fact that we have an inner action and using Definition 2.2, $\mathbb{1}_{\mathcal{A}}$ is clear. Since $\mathrm{a}_{J}$ takes values in the center only, one easily computes $\mathrm{a}_{J}(g h)=\mathrm{a}_{J}(g) \mathrm{a}_{J}(h)$ using the property ii. ) from Definition 2.2. It follows that $\mathrm{a}_{J}$ is a unital homomorphism into $\mathfrak{Z}(\mathcal{A})$. Note that $J$ has a convolution inverse given by $J^{-1}(g)=J(S(g))$ since it is a unital homomorphism. From this and (2.5) we see that $J^{*}=J^{-1}$. It easily follows that (4.3) maps convolution products to convolution products and hence gives a group morphism into $\mathrm{Gl}(H, \mathfrak{Z}(\mathcal{A}))$. Finally, $\left(\mathrm{a}^{*}\right)_{J}=\left(\mathrm{a}_{J}\right)^{*}$ holds which shows that $\mathrm{a}_{J} \in \mathrm{U}(H, \mathfrak{Z}(\mathcal{A}))$ for a $\in \mathrm{U}(H, \mathcal{A})$. The injectivity of (4.3) is clear, we only have to check surjectivity. Thus let $\mathrm{z} \in \mathrm{U}(H, \mathfrak{Z}(\mathcal{A}))$ be given and consider the pre-image $\mathrm{a}=J * \mathrm{z} * J^{-1}$ under (4.3) in $\operatorname{Hom}_{\mathrm{C}}(H, \mathcal{A})$. Clearly, $\mathrm{a}\left(\mathbb{1}_{H}\right)=\mathbb{1}_{\mathcal{A}}$ holds. Using the facts that $\mathrm{z}$ takes values in the center and that the action is inner allows to show the properties [ii.) and iii.) for a by a simple computation. Thus $\mathrm{a} \in \mathrm{Gl}(H, \mathcal{A})$ follows. Finally, from $\mathrm{z}^{-1}=\mathrm{z}^{*}$ and the compatibility of (4.3) with the ${ }^{*}$-involution (2.5) we conclude that $a \in \mathrm{U}(H, \mathcal{A})$ as wanted. This concludes the proof as the ring-theoretic part was done on the way as well.

The next lemma characterizes the uniqueness properties of momentum maps which lead to the same action:

Lemma 4.3 Let $\mathcal{A}$ be a unital ${ }^{*}$-algebra with ${ }^{*}$-action of a Hopf ${ }^{*}$-algebra $H$. For any two momentum maps $J, J^{\prime} \in \operatorname{MoMa}_{H}^{*}(\mathcal{A})$ the map $\mathbf{z}=J^{-1} * J^{\prime}$ defines an element $\mathbf{z} \in \mathrm{U}(H, \mathfrak{Z}(\mathcal{A}))$. Conversely, for $J \in \operatorname{MoMa}_{H}^{*}(\mathcal{A})$ and $\mathrm{z} \in \mathrm{U}(H, \mathfrak{Z}(\mathcal{A}))$ we have $J * \mathrm{z} \in \operatorname{MoMa}_{H}^{*}(\mathcal{A})$.

Proof. Let $a \in \mathcal{A}$ then we compute

$$
\mathrm{z}(g) a=J\left(S\left(g_{(1)}\right)\right) J^{\prime}\left(g_{(2)}\right) a=J\left(S\left(g_{(1)}\right)\right)\left(g_{(2)} \triangleright a\right) J^{\prime}\left(g_{(3)}\right)=a J\left(S\left(g_{(1)}\right)\right) J^{\prime}\left(g_{(2)}\right)=a \mathrm{z}(g)
$$

showing $\mathbf{z}(g) \in \mathfrak{Z}(\mathcal{A})$ for all $g \in H$. Using (4.2), the remaining properties for $\mathbf{z} \in \mathbf{U}(H, \mathfrak{Z}(\mathcal{A}))$ are easily verified and the converse statement follows directly.

The ring-theoretic situation is handled analogously replacing $\mathrm{U}(H, \mathfrak{Z}(\mathcal{A}))$ by $\mathrm{Gl}(H, \mathfrak{Z}(\mathcal{A}))$ : we do not need to formulate this in detail. In the case of $\left(C^{\infty}(M)[[\lambda]], \star\right)$ and $\mathcal{U}_{\lambda}(\mathfrak{g})$, the ambiguity in the existence of quantum momentum maps reduces to the one in [20], see also the discussion in [22].

Since $\mathrm{U}(H, \mathfrak{Z}(\mathcal{A}))$ and $\mathrm{U}(H, \mathcal{A})$ are isomorphic via (4.3) we can also parametrize momentum maps $J^{\prime}$ starting with a fixed reference momentum map $J$ and a $\in \mathrm{U}(H, \mathcal{A})$ by $J^{\prime}=J * \mathrm{z}=\mathrm{a} * J$ where $\mathbf{z}=\mathrm{a}_{J} \in \mathrm{U}(H, \mathfrak{Z}(\mathcal{A}))$. We summarize these results in the following theorem:

Theorem 4.4 Let $\mathcal{A}$ be a unital ${ }^{*}$-algebra with ${ }^{*}$-action of $H$ and a momentum map $J$. Then the group $\mathrm{U}(H, \mathcal{A})$ acts freely and transitively from the left on $\mathrm{MoMa}_{H}^{*}(\mathcal{A})$ by left multiplication using the convolution product $*$ of $\operatorname{Hom}_{\mathrm{C}}(H, \mathcal{A})$. Equivalently, $\mathrm{U}(H, \mathfrak{Z}(\mathcal{A}))$ acts freely and transitively on $\operatorname{MoMa}_{H}^{*}(\mathcal{A})$ from the right by the convolution product. In the ring-theoretic situation, $\operatorname{MoMa}_{H}^{*}(\mathcal{A})$, $\mathrm{U}(H, \mathcal{A})$, and $\mathrm{U}(H, \mathfrak{Z}(\mathcal{A}))$ have to be replaced by $\operatorname{MoMa}_{H}(\mathcal{A}), \operatorname{Gl}(H, \mathcal{A})$, and $\mathrm{Gl}(H, \mathfrak{Z}(\mathcal{A}))$, respectively. 


\subsection{The splitting}

The following consideration is central. We state the result in slightly larger generality though we need this only for equivalence bimodules later.

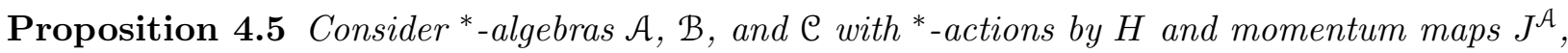
$J^{\mathcal{B}}$, and $J^{\mathcal{C}}$, respectively.

i.) If ${ }_{\mathcal{B}} \mathcal{E}_{\mathcal{A}}$ is a $(\mathcal{B}, \mathcal{A})$-bimodule then

$$
h \triangleright x=J^{\mathcal{B}}\left(h_{(1)}\right) \cdot x \cdot J^{\mathcal{A}}\left(S\left(h_{(2)}\right)\right)
$$

for $h \in H$ and $x \in{ }_{\mathcal{B}} \mathcal{E}_{\mathcal{A}}$ defines an action of $H$ on ${ }_{\mathcal{B}} \mathcal{E}_{\mathcal{A}}$ which is compatible with the bimodule structure.

ii.) Endowing two $(\mathcal{B}, \mathcal{A})$-bimodules ${ }_{\mathcal{B}} \mathcal{E}_{\mathcal{A}}$ and ${ }_{\mathcal{B}} \mathcal{E}_{\mathcal{A}}^{\prime}$ with this $H$-action makes every bimodule morphism $\Phi:{ }_{\mathcal{B}} \mathcal{E}_{\mathcal{A}} \longrightarrow{ }_{\mathcal{B}} \mathcal{E}_{\mathcal{A}}^{\prime}$ equivariant with respect to the $H$-actions (4.4).

iii.) If a $(\mathcal{B}, \mathcal{A})$-bimodule ${ }_{\mathcal{B}} \mathcal{E}_{\mathcal{A}}$ carries an algebra-valued inner product then the action (4.4) is compatible with it.

iv.) For a $(\mathcal{C}, \mathcal{B})$-bimodule ${ }_{\mathfrak{e}} \mathcal{F}_{\mathcal{B}}$ and a $(\mathcal{B}, \mathcal{A})$-bimodule ${ }_{\mathcal{B}} \mathcal{E}_{\mathcal{A}}$ the lift (4.4) to ${ }_{\mathrm{e}} \mathcal{F}_{\mathcal{B}} \otimes_{\mathcal{B}} \mathcal{E}_{\mathcal{A}}$ coincides with the tensor product of the lifts to ${ }_{\mathrm{e}} \mathcal{F}_{\mathcal{B}}$ and ${ }_{\mathcal{B}} \varepsilon_{\mathcal{A}}$.

v.) For the canonical bimodule ${ }_{\mathcal{A}} \mathcal{A}_{\mathcal{A}}$ the lift (4.4) reproduces the original action of $H$ on $\mathcal{A}$.

Proof. It follows immediately that (4.4) yields a compatible $H$-action on the bimodule ${ }_{\mathcal{B}} \mathcal{E}_{\mathcal{A}}$. Being an "inner" action the second statement is trivial. The compatibility with either a $\mathcal{B}$-valued or an $\mathcal{A}$-valued inner product is a direct calculation

$$
\begin{aligned}
h \triangleright\langle x, y\rangle_{\mathcal{A}} & =J^{\mathcal{A}}\left(h_{(1)}\right)\langle x, y\rangle_{\mathcal{A}} J^{\mathcal{A}}\left(S\left(h_{(2)}\right)\right) \\
& =J^{\mathcal{A}}\left(h_{(1)}\right)\left\langle J^{\mathcal{B}}\left(S\left(h_{(2)}\right)^{*}\right) \cdot x, J^{\mathcal{B}}\left(h_{(3)}\right) \cdot y\right\rangle_{\mathcal{A}} J^{\mathcal{A}}\left(S\left(h_{(4)}\right)\right) \\
& =\left\langle J^{\mathcal{B}}\left(S\left(h_{(2)}\right)^{*}\right) \cdot x \cdot J^{\mathcal{A}}\left(S\left(S\left(h_{(1)}\right)^{*}\right)\right), J^{\mathcal{B}}\left(h_{(3)}\right) \cdot y \cdot J^{\mathcal{A}}\left(S\left(h_{(4)}\right)\right)\right\rangle_{\mathcal{A}} \\
& =\left\langle S\left(h_{(1)}\right)^{*} \triangleright x, h_{(2)} \triangleright y\right\rangle_{\mathcal{A}} .
\end{aligned}
$$

The compatibility with the tensor product follows from

$$
\begin{aligned}
\left(h_{(1)} \triangleright x\right) \otimes_{\mathcal{B}}\left(h_{(2)} \triangleright y\right) & =\left(J^{\mathcal{C}}\left(h_{(1)}\right) \cdot x \cdot J^{\mathcal{B}}\left(S\left(h_{(2)}\right)\right)\right) \otimes_{\mathcal{B}}\left(J^{\mathcal{B}}\left(h_{(3)}\right) \cdot y \cdot J^{\mathcal{A}}\left(S\left(h_{(4)}\right)\right)\right) \\
& =\left(J^{\mathcal{C}}\left(h_{(1)}\right) \cdot x\right) \otimes_{\mathcal{B}}\left(y \cdot J^{\mathcal{A}}\left(h_{(2)}\right)\right) \\
& =h \triangleright\left(x \otimes_{\mathcal{B}} y\right) .
\end{aligned}
$$

The last part is clear.

Note that the proposition also holds in the ring-theoretic case, except of course for the statement about the inner products.

In the following, we consider $H$-equivariant Morita theory only for ${ }^{*}$-algebras with a specific momentum map. In particular, we consider the Picard groupoids of the various flavours only over this restricted class of algebras. From the above Proposition 4.5 it follows that for every *- or strong equivalence bimodule ${ }_{\mathcal{B}} \mathcal{E}_{\mathcal{A}}$ the lift (4.4) endows ${ }_{\mathcal{B}} \mathcal{E}_{\mathcal{A}}$ with the structure of an $H$-equivariant *- or strong equivalence bimodule, respectively. Moreover, the compatibility with tensor products and 
morphisms guarantees that we obtain a groupoid morphism after passing to isomorphism classes. We denote these groupoid morphisms by

$$
J: \mathrm{Pic}^{*} \longrightarrow \mathrm{Pic}_{H}^{*} \quad \text { and } \quad J: \mathrm{Pic}^{\text {str }} \longrightarrow \mathrm{Pic}_{H}^{\text {str }}
$$

respectively. Note that we suppress the restriction to this more specific class of *-algebras in our notation. Analogously, in the ring-theoretic situation we get a groupoid morphism

$$
J: \mathrm{Pic} \longrightarrow \mathrm{Pic}_{H}
$$

Remark 4.6 In fact, the above proposition even gives a functor $J$ : Bimod $\longrightarrow$ Bimod $_{\mathrm{H}}$ whose restriction to invertible arrows is (4.6) and similarly for the ${ }^{*}$ - and strong version. However, we will not need this additional structure.

The following theorem is now an easy consequence of these considerations which we formulate for all three versions of the Picard groupoid:

Theorem 4.7 Consider the Picard groupoids over the restricted class of unital ${ }^{*}$-algebras (or algebras) with momentum maps.

i.) The groupoid morphisms $J$ from (4.5) and (4.6), respectively, are right inverses of the canonical groupoid morphisms in (2.3). In particular, over this class, (2.3) is surjective.

ii.) Let $\mathcal{A}$ be fixed unital ${ }^{*}$-algebra (or algebra) with momentum map. The images of the subgroups $\mathrm{GL}(\mathfrak{Z}(\mathcal{A}))$ and $\mathrm{U}(\mathfrak{Z}(\mathcal{A}))$ in $\mathrm{Gl}(H, \mathcal{A})$ and $\mathrm{U}(H, \mathcal{A})$, respectively, under the morphism (2.8) are trivial. Hence we identify $\mathrm{Gl}_{0}(H, \mathcal{A})=\mathrm{Gl}(H, \mathcal{A})$ and $\mathrm{U}_{0}(H, \mathcal{A})=\mathrm{U}(H, \mathcal{A})$.

iii.) For a fixed unital ${ }^{*}$-algebra (or algebra) with momentum map there are split exact sequences

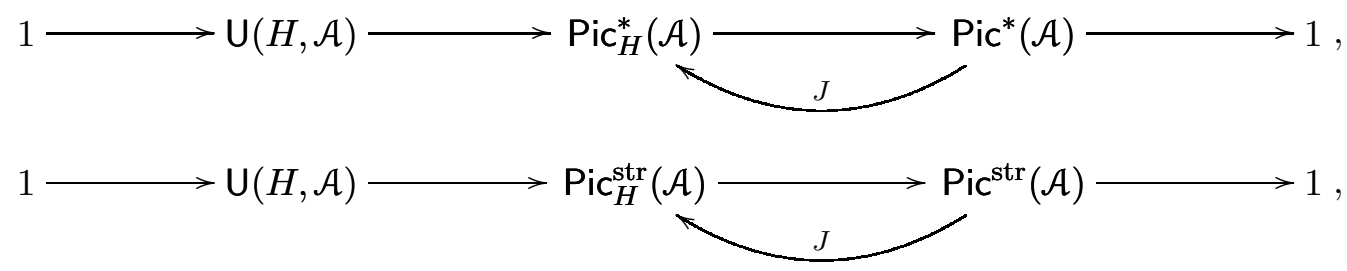

and

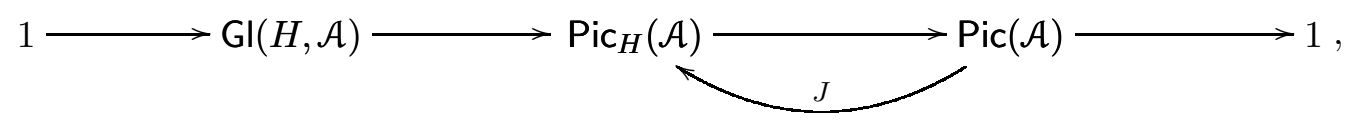

respectively.

iv.) For a fixed unital ${ }^{*}$-algebra (or algebra) with momentum map the H-equivariant Picard groups are semidirect products

$$
\operatorname{Pic}_{H}^{*}(\mathcal{A}) \cong \operatorname{Pic}^{*}(\mathcal{A}) \ltimes \mathrm{U}(H, \mathcal{A}) \quad \text { and } \quad \operatorname{Pic}_{H}^{\operatorname{str}}(\mathcal{A}) \cong \operatorname{Pic}^{\operatorname{str}}(\mathcal{A}) \ltimes \mathrm{U}(H, \mathcal{A}),
$$

as well as

$$
\operatorname{Pic}_{H}(\mathcal{A}) \cong \operatorname{Pic}(\mathcal{A}) \ltimes \mathrm{Gl}(H, \mathcal{A})
$$


respectively. The group actions responsible for the semidirect product are induced by

$$
\Phi(\mathcal{E}): \mathrm{U}(H, \mathcal{A}) \ni \mathrm{a} \longmapsto h_{J(\mathcal{E})}(\mathrm{a}) \in \mathrm{U}(H, \mathcal{A})
$$

for the *- and strong version with $h_{J(\mathcal{E})}$ as in Lemma 2.4, and by

$$
\Phi(\mathcal{E}): \mathrm{Gl}(H, \mathcal{A}) \ni \mathrm{a} \longmapsto h_{J(\mathcal{E})}(\mathrm{a}) \in \mathrm{GI}(H, \mathcal{A})
$$

in the ring-theoretic framework, respectively.

Proof. The first part is clear as forgetting the lifted action reproduces the bimodule we started with. Proposition 4.5 ensures that $J$ is indeed a groupoid morphism. The second part follows from $\mathfrak{Z}(\mathcal{A}) \subseteq \mathcal{A}^{H}$ for an algebra with an inner $H$-action. The third part follows from (2.9) and (2.10), respectively, and the groupoid structure at once. For the last part we have to compute the precise action of the Picard group on the groups $\mathrm{U}(H, \mathcal{A})$ and $\mathrm{Gl}(H, \mathcal{A})$, respectively. Thus let ${ }_{\mathcal{A}} \mathcal{E}_{\mathcal{A}}$ be a ${ }^{*}$ equivalence $(\mathcal{A}, \mathcal{A})$-bimodule and $\mathrm{a} \in \mathrm{U}(H, \mathcal{A})$. In order to compute the action of ${ }_{\mathcal{A}} \varepsilon_{\mathcal{A}}$ on a we have to consider the *-equivalence bimodule ${ }_{\mathcal{A}} \varepsilon_{\mathcal{A}} \otimes{ }_{\mathcal{A}} \mathcal{A}_{\mathcal{A}} \otimes{ }_{\mathcal{A}} \bar{\varepsilon}_{\mathcal{A}} \cong{ }_{\mathcal{A}} \mathcal{A}_{\mathcal{A}}$. When ${ }_{\mathcal{A}} \mathcal{E}_{\mathcal{A}}$ is equipped with the lifted action we have to determine the action $\triangleright^{\Phi(\mathcal{E})(\mathrm{a})}$ on this tensor product when ${ }_{\mathcal{A}} \mathcal{A}_{\mathcal{A}}$ was endowed with the twisted action $\triangleright^{a}$. But this is now an easy computation as the action $\triangleright^{\Phi(\mathcal{E})(\mathrm{a})}$ is given by the tensor product of the actions on each factor, respectively. Let $x \otimes a \otimes \bar{y} \in J(\mathcal{E}) \otimes \mathcal{A} \otimes J(\overline{\mathcal{E}})$. Then

$$
\begin{aligned}
g \triangleright^{\Phi(\mathcal{E})(\mathrm{a})}(x \otimes a \otimes \bar{y}) & =\left(g_{(1)} \triangleright x\right) \otimes\left(g_{(2)} \triangleright^{\mathrm{a}} a\right) \otimes\left(g_{(3)} \triangleright \bar{y}\right) \\
& =\left(g_{(1)} \triangleright x\right) \otimes\left(\mathrm{a}\left(g_{(2)}\right) \cdot\left(g_{(3)} \triangleright a\right)\right) \otimes\left(g_{(4)} \triangleright \bar{y}\right) \\
& =\left(\left(g_{(1)} \triangleright x\right) \cdot \mathrm{a}\left(g_{(2)}\right)\right) \otimes\left(g_{(3)} \triangleright a\right) \otimes\left(g_{(4)} \triangleright \bar{y}\right) \\
& =\left(g_{(1)} \triangleright_{\mathrm{a}} x\right) \otimes\left(g_{(2)} \triangleright a\right) \otimes\left(g_{(3)} \triangleright \bar{y}\right) \\
& =\left(g_{(1)} \triangleright^{h_{J(\varepsilon)}}(\mathrm{a})\right. \\
& x) \otimes\left(g_{(2)} \triangleright a\right) \otimes\left(g_{(3)} \triangleright \bar{y}\right) \\
& =g \triangleright^{h_{J(\varepsilon)}(\mathrm{a})}(x \otimes a \otimes \bar{y}) .
\end{aligned}
$$

Thus, $\Phi(\mathcal{E})(\mathrm{a})=h_{J(\varepsilon)}$ (a) follows. The ring-theoretic case is analogous.

On the other hand, the existence of a momentum map is an invariant for equivariant Morita equivalence. This shows that our restriction to the above class is not severe: all other unital * algebras (algebras) with other types of *-actions (actions) of $H$ are necessarily on different connected components of the corresponding Picard groupoids and hence "invisible" from the Morita theory point of view. In [16], Morita invariants are seen as arising from actions of the corresponding Picard groupoids. We follow this point of view and formulate this Morita invariant by means of an action. To this end we need the following technical lemma:

Lemma 4.8 Consider unital ${ }^{*}$-algebras $\mathcal{A}, \mathcal{B}$, and $\mathcal{C}$ with $a^{*}$-action of the Hopf ${ }^{*}$-algebra $H$. Furthermore, assume that $\operatorname{MoMa}_{H}^{*}(\mathcal{A}) \neq \emptyset$.

i.) Assume there is an $H$-equivariant ${ }^{*}$-equivalence bimodule ${ }_{\mathcal{B}} \mathcal{E}_{\mathcal{A}}$. For $J \in \mathrm{MoMa}_{H}^{*}(\mathcal{A})$ there exists a uniquely determined momentum map $h_{\mathcal{E}}(J) \in \mathrm{MoMa}_{H}^{*}(\mathcal{B})$ such that for all $g \in H$ and $x \in{ }_{\mathcal{B}} \mathcal{E}_{\mathcal{A}}$

$$
g \triangleright x=h_{\mathcal{E}}(J)\left(g_{(1)}\right) \cdot x \cdot J\left(S\left(g_{2}\right)\right) .
$$

ii.) Assume ${ }_{B} \mathcal{E}_{\mathcal{A}},{ }_{B} \mathcal{E}_{\mathcal{A}}^{\prime}$ are isomorphic $H$-equivariant ${ }^{*}$-equivalence bimodules. Then

$$
h_{\mathcal{E}}=h_{\mathcal{E}^{\prime}} .
$$


iii.) Assume ${ }_{\mathcal{B}} \mathcal{E}_{\mathcal{A}}$ and ${ }_{\mathrm{e}} \mathcal{F}_{\mathcal{B}}$ are $H$-equivariant ${ }^{*}$-equivalence bimodules. Then

$$
h_{\mathcal{F}} \circ h_{\mathcal{E}}=h_{\mathcal{F} \otimes \mathcal{E}} \quad \text { and } \quad h_{\mathcal{A}}=\operatorname{id}_{\operatorname{MoMa}_{H}^{*}(\mathcal{A})} .
$$

iv.) Assume ${ }_{\mathcal{B}} \mathcal{E}_{\mathcal{A}}$ is an $H$-equivariant ${ }^{*}$-equivalence bimodule. Then for a $\in \mathrm{U}(H, \mathcal{A})$ and $J \in$ $\operatorname{MoMa}_{H}^{*}(\mathcal{A})$ we have

$$
h_{\mathcal{E}}(\mathrm{a} * J)=h_{\mathcal{E}}(\mathrm{a}) * h_{\mathcal{E}}(J) .
$$

Proof. For the first part, let $a \in \mathcal{A}$ and $x \in{ }_{\mathcal{B}} \mathcal{E}_{\mathcal{A}}$. Then for $g \in H$ we have

$$
\begin{aligned}
\left(g_{(1)} \triangleright(x \cdot a)\right) \cdot J\left(g_{(2)}\right) & =\left(g_{(1)} \triangleright x\right) \cdot\left(\left(g_{(2)} \triangleright a\right) J\left(g_{(3)}\right)\right) \\
& =\left(g_{(1)} \triangleright x\right) \cdot\left(J\left(g_{(2)}\right) a J\left(S\left(g_{(3)}\right)\right) J\left(g_{(4)}\right)\right) \\
& =\left(\left(h_{(1)} \triangleright x\right) \cdot J\left(h_{(2)}\right)\right) \cdot a .
\end{aligned}
$$

This shows that the map $x \mapsto\left(g_{(1)} \triangleright x\right) \cdot J\left(g_{(2)}\right)$ is right $\mathcal{A}$-linear and depends linearly on $g$. Thus it is the left multiplication by a unique element $h_{\mathcal{E}}(J)(g) \in \mathcal{B}$, which depends linearly on $g$. We claim that this defines the momentum map we are looking for. First we have for $g, h \in H$ and $x \in{ }_{\mathcal{B}} \mathcal{E}_{\mathcal{A}}$

$$
\left(h_{\mathcal{E}}(J)(g) h_{\mathcal{E}}(J)(h)\right) \cdot x=h_{\mathcal{E}}(J)(g h) \cdot x,
$$

by simply expanding the definition. Moreover, $h_{\mathcal{E}}(J)\left(\mathbb{1}_{H}\right)=\mathbb{1}_{\mathcal{B}}$ is clear. It is now a simple check that (4.14) holds. Let $b \in \mathcal{B}$ and $x \in{ }_{\mathcal{B}} \mathcal{E}_{\mathcal{A}}$ be given. Then

$$
\begin{aligned}
(g \triangleright b) \cdot x & =g_{(1)} \triangleright\left(b \cdot\left(S\left(g_{(2)}\right) \triangleright x\right)\right) \\
& =h_{\mathcal{E}}(J)\left(g_{(1)}\right) \cdot\left(b \cdot\left(h_{\mathcal{E}}(J)\left(S\left(g_{(3)}\right)_{(1)}\right) \cdot x \cdot J\left(S\left(S\left(g_{(3)}\right)_{(2)}\right)\right)\right)\right) \cdot J\left(S\left(g_{(2)}\right)\right) \\
& =\left(h_{\mathcal{E}}(J)\left(g_{(1)}\right) b h_{\mathcal{E}}(J)\left(S\left(g_{(4)}\right)\right)\right) \cdot x \cdot\left(J\left(S^{2}\left(g_{(3)}\right)\right) J\left(S\left(g_{(2)}\right)\right)\right) \\
& =\left(h_{\mathcal{E}}(J)\left(g_{(1)}\right) b h_{\mathcal{E}}(J)\left(S\left(g_{(4)}\right)\right)\right) \cdot x \cdot\left(J\left(S\left(g_{(2)} S\left(g_{(3)}\right)\right)\right)\right) \\
& =\left(h_{\mathcal{E}}(J)\left(g_{(1)}\right) b h_{\mathcal{E}}(J)\left(S\left(g_{(2)}\right)\right)\right) \cdot x
\end{aligned}
$$

shows that $h_{\mathcal{E}}(J)$ is a momentum map for the algebra $\mathcal{B}$. Finally, we use the compatibility of the $\mathcal{A}$-valued inner product on ${ }_{\mathcal{B}} \mathcal{E}_{\mathcal{A}}$ with the action and compute for $x, y \in{ }_{\mathcal{B}} \mathcal{E}_{\mathcal{A}}$ and $g \in H$

$$
\begin{aligned}
\left\langle h_{\mathcal{E}}(J)(g)^{*} \cdot x, y\right\rangle_{\mathcal{A}} & =\left\langle x, h_{\mathcal{E}}(J) \cdot y\right\rangle_{\mathcal{A}} \\
& =\left\langle x,\left(g_{(1)} \triangleright y\right) \cdot J\left(g_{(2)}\right)\right\rangle_{\mathcal{A}} \\
& =\left(g_{(2)} \triangleright\left\langle g_{(1)}^{*} \triangleright x, y\right\rangle_{\mathcal{A}}\right) J\left(g_{(3)}\right) \\
& =J\left(g_{(2)}\right)\left\langle g_{(1)}^{*} \triangleright x, y\right\rangle_{\mathcal{A}} \\
& =\left\langle\left(g_{(1)}^{*} \triangleright x\right) \cdot J\left(g_{(2)}^{*}\right), y\right\rangle_{\mathcal{A}} \\
& =\left\langle h_{\mathcal{E}}(J)\left(g^{*}\right) \cdot x, y\right\rangle_{\mathcal{A}} .
\end{aligned}
$$

Since the inner product is non-degenerate this establishes $h_{\mathcal{E}}(J)(g)^{*}=h_{\mathcal{E}}(J)\left(g^{*}\right)$. Thus $h_{\mathcal{E}}(J)$ is indeed a momentum map for the ${ }^{*}$-algebra $\mathcal{B}$. Finally, the way we constructed $h_{\mathcal{E}}(J)$ gives the uniqueness. This completes the first part. For the second part, let $\Psi:{ }_{\mathcal{B}} \mathcal{E}_{\mathcal{A}} \longrightarrow{ }_{\mathcal{B}} \mathcal{E}_{\mathcal{A}}^{\prime}$ be an $H$-equivariant isometric isomorphism. Then

$$
g \triangleright x=\Psi^{-1}(g \triangleright \Psi(x))=\Psi^{-1}\left(h_{\mathcal{E}^{\prime}}(J)\left(g_{(1)}\right) \cdot \Psi(x) \cdot J\left(S\left(g_{(2)}\right)\right)\right)=h_{\mathcal{E}^{\prime}}(J)\left(g_{(1)}\right) \cdot x \cdot J\left(S\left(g_{(2)}\right)\right)
$$

by equivariance and the fact that $\Psi$ is a bimodule isomorphism. This shows that $h_{\mathcal{E}^{\prime}}(J)$ satisfies (4.14) and thus coincides with $h_{\mathcal{E}}(J)$ by uniqueness. For the third part, let ${ }_{e} \mathcal{F}_{\mathcal{B}}$ be yet another 
$H$-equivariant ${ }^{*}$-equivalence bimodule. For factorizing tensors $y \otimes x \in{ }_{\mathcal{e}} \mathcal{F}_{\mathcal{B}} \otimes_{\mathcal{B}} \mathcal{E}_{\mathcal{A}}$ we unwind the definition

$$
\begin{aligned}
h_{\mathcal{F} \otimes \mathcal{E}}(J) \cdot(y \otimes x) & =\left(g_{(1)} \triangleright(y \otimes x)\right) \cdot J\left(g_{(2)}\right) \\
& =\left(g_{(1)} \triangleright y\right) \otimes\left(\left(g_{(2)} \triangleright x\right) \cdot J\left(g_{(3)}\right)\right) \\
& =\left(g_{(1)} \triangleright y\right) \otimes\left(h_{\mathcal{E}}(J)\left(g_{(2)}\right) \cdot x\right) \\
& =h_{\mathcal{F}}\left(h_{\mathcal{E}}(J)\right)(g) \cdot(y \otimes x),
\end{aligned}
$$

showing the first claim in (4.16). The second statement in (4.16) is trivial. For the last part we consider $\mathrm{a} \in \mathrm{U}(H, \mathcal{A})$ and compute for $x \in{ }_{\mathcal{B}} \mathcal{E}_{\mathcal{A}}$

$$
\begin{aligned}
h_{\mathcal{E}}(\mathrm{a} * J) \cdot x & =\left(g_{(1)} \triangleright x\right) \cdot\left(\mathrm{a}\left(g_{(2)}\right) J\left(g_{(3)}\right)\right) \\
& =\left(g_{(1)} \triangleright_{\mathrm{a}} x\right) \cdot J\left(g_{(2)}\right) \\
& =\left(g_{(1)} \triangleright_{\mathcal{E}}(\mathrm{a}) x\right) \cdot J\left(g_{(2)}\right) \\
& =h_{\mathcal{E}}(\mathrm{a})\left(g_{(1)}\right) \cdot\left(\left(g_{(2)} \triangleright x\right) \cdot J\left(g_{(3)}\right)\right) \\
& =\left(h_{\mathcal{E}}(\mathrm{a})\left(g_{(1)}\right) h_{\mathcal{E}}(J)\left(g_{(2)}\right)\right) \cdot x,
\end{aligned}
$$

using Lemma 2.4. This shows (4.17).

Rephrasing the above lemma gives us the following general statement on the existence of momentum maps:

Theorem 4.9 ( $\mathrm{Pic}_{H}^{*}$ acts on $\left.\mathrm{MoMa}_{H}^{*}\right)$ The H-equivariant ${ }^{*}$-Picard groupoid $\mathrm{Pic}_{H}^{*}$ acts on the $\mathrm{U}(H, \cdot)$-spaces $\mathrm{MoMa}_{H}^{*}(\cdot)$ from the left. In particular, the existence of a momentum map is invariant under $H$-equivariant ${ }^{*}$-Morita equivalence.

Here it is understood that the groupoid action of $\mathrm{Pic}_{H}^{*}$ on $\mathrm{MoMa}_{H}^{*}(\cdot)$ is "along" the groupoid action of $\mathrm{Pic}_{H}^{*}$ on $\mathrm{U}(H, \cdot)$ from Lemma 2.4. In formulas, this just means (4.17).

Dropping the *-involutions gives us the ring-theoretic result which means that the $H$-equivariant Picard groupoid acts on the $\mathrm{Gl}(H, \cdot)$-spaces $\operatorname{MoMa}_{H}(\cdot)$ from the left. Again, the existence of a momentum map is invariant under $H$-equivariant Morita equivalence.

In the sequel, we will need more precisely the structure of $\operatorname{Pic}_{H}^{*}(\mathcal{A})$ for a commutative unital *-algebra $\mathcal{A}$. In this case, we do, of course, not assume to have an inner action.

Lemma 4.10 Let $\mathcal{A}$ and $\mathcal{B}$ be commutative unital ${ }^{*}$-algebras and let $\left[{ }_{B_{\mathcal{B}}} \mathcal{E}_{\mathcal{A}}\right] \in \operatorname{Pic}_{H}^{*}(\mathcal{B}, \mathcal{A})$. Then there is an $H$-equivariant ${ }^{*}$-isomorphism $h_{\mathcal{E}}: \mathcal{A} \longrightarrow \mathcal{B}$ determined by $h_{\mathcal{E}}(a) \cdot x=x \cdot a$. The map $\mathcal{E} \mapsto h_{\mathcal{E}}$ is compatible with the composition and units in $\mathrm{Pic}_{H}^{*}$.

Proof. This is [16, Lemma 5.1], except for the $H$-equivariance of $h_{\varepsilon}$ which is easy to see.

Denote by $\operatorname{Aut}_{H}^{*}(\mathcal{A})$ the $H$-equivariant *-automorphism of the *-algebra $\mathcal{A}$ and by $\operatorname{SPic}_{H}^{*}(\mathcal{A})$ the equivalence classes of static $H$-equivariant bimodules in $\operatorname{Pic}_{H}^{*}(\mathcal{A})$. Recall that a $(\mathcal{A}, \mathcal{A})$-bimodule ${ }_{\mathcal{A}} \mathcal{E}_{\mathcal{A}}$ is called static (or commutative or central), if $a \cdot x=x \cdot a$ for all $a \in \mathcal{A}$ and $x \in{ }_{\mathcal{A}} \mathcal{E}_{\mathcal{A}}$. Analogously, one defines $\operatorname{SPic}_{H}^{\mathrm{str}}(\mathcal{A})$ and the ring-theoretic version $\operatorname{SPic}_{H}(\mathcal{A})$.

Proposition 4.11 For a commutative unital ${ }^{*}$-algebra $\mathcal{A}$ with ${ }^{*}$-action of $H$ one has

$$
\operatorname{Pic}_{H}^{*}(\mathcal{A}) \cong \operatorname{Aut}_{H}^{*}(\mathcal{A}) \ltimes \operatorname{SPic}_{H}^{*}(\mathcal{A}),
$$

and analogously for $\operatorname{Pic}_{H}^{\mathrm{str}}(\mathcal{A})$. In the ring-theoretic case we have $\operatorname{Pic}_{H}(\mathcal{A}) \cong \operatorname{Aut}_{H}(\mathcal{A}) \ltimes \operatorname{SPic}_{H}(\mathcal{A})$. 
Proof. For an ${ }^{*}$-automorphism $\Phi \in \operatorname{Aut}^{*}(\mathcal{A})$ we can twist the canonical strong self-equivalence ${ }_{\mathcal{A}} \mathcal{A}_{\mathcal{A}}$ by modifying the left module structure to $a \cdot_{\Phi} x=\Phi^{-1}(a) x$. The right module structure is kept unchanged and the inner products are changed appropriately. This gives a group morphism $\ell: \operatorname{Aut}^{*}(\mathcal{A}) \longrightarrow \operatorname{Pic}^{\operatorname{str}}(\mathcal{A})$. It turns out that this is compatible with the $H$-equivariance if $\Phi$ is $H$ equivariant. In fact, this construction comes from a groupoid morphism $\ell$ from the $H$-equivariant *-isomorphism groupoid Iso $_{H}^{*}$ to $\mathrm{Pic}_{H}^{\text {str }}$, see [16, Prop. 4.7]. If now $\mathcal{A}$ is commutative $\operatorname{SPic}_{H}^{\text {str }}(\mathcal{A})$ can be viewed as a normal subgroup of $\operatorname{Pic}_{H}^{\operatorname{str}}(\mathcal{A})$ and with the map $h$ from Lemma 4.10 we get an exact sequence

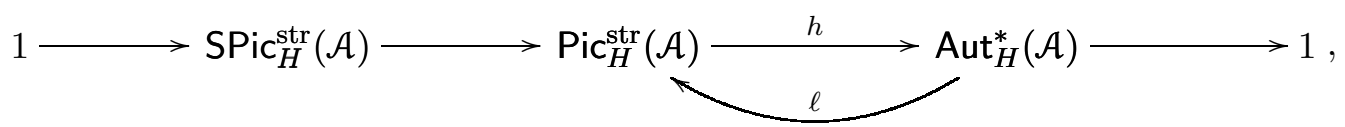

which splits by $\ell$. The case of *-equivalence is analogous and the ring-theoretic case is even simpler. Note that the above split exact sequence is classic for the non-equivariant, ring-theoretic case, see e.g. [2, Chap. II, Prop. 5.4].

\section{Equivariant Morita equivalence of star products}

We will now apply the notion of equivariant Morita equivalence of algebras to star products on $(M, \omega)$. The setting is again as in Section 3 and we will call the $\mathcal{U}_{\mathbb{C}}(\mathfrak{g})[[\lambda]]$-equivariance simply $\mathfrak{g}$-equivariance throughout this section.

\subsection{Classical and semiclassical limits of a g-equivariant equivalence bimodule}

In the sequel we want to explore the classical and semiclassical limit of equivalence bimodules with respect to the symmetry structures. We have to take care of the "up to isomorphism" statements as in Section 3 more carefully. Recall that the classical limit of an equivalence bimodule $\mathcal{L}$ for two star products is defined by the $C^{\infty}(M)$-bimodule

$$
\operatorname{cl}(\mathcal{L})=\mathcal{L} / \lambda \mathcal{L}
$$

and yields an equivalence bimodule for $C^{\infty}(M)$. The quotient map is also referred to as the classical limit and will be denoted by cl. Furthermore, one has the following result, see e.g. [8]:

Proposition 5.1 Let $\mathcal{L}$ be an equivalence bimodule for the two star products $\star$ and $\star^{\prime}$ where $\star^{\prime}$ acts from the left. Then there exists a unique symplectomorphism $\Psi$ such that ${ }^{\Psi} \mathcal{L}$ with the twisted left module structure by $\Psi$ is an equivalence bimodule for $\Psi^{*}\left(\star^{\prime}\right)$ and $\star$ and $\operatorname{cl}\left({ }^{\Psi} \mathcal{L}\right)$ is a static equivalence bimodule for $C^{\infty}(M)$ and hence isomorphic to the sections $\mathcal{L}=\Gamma^{\infty}(L)$ of a line bundle $L$.

Thus we can replace $\star^{\prime}$ by $\Psi^{*}\left(\star^{\prime}\right)$ and assume that the equivalence bimodule has a static equivalence bimodule as classical limit. A further result from the general ring-theoretic deformation theory is that in this case where $\mathcal{L}$ has a static classical limit $\mathcal{L}$ we have a $\mathbb{C}[[\lambda]]$-linear isomorphism $\mathcal{L} \cong \mathcal{L}[[\lambda]]$ which induces a bimodule deformation of $\mathcal{L}$ encoded by the deformed left and right multiplication laws $\bullet$ and $\bullet$ as in (3.4) and (3.2), respectively. Thus it will be sufficient to assume that $\mathcal{L}=\left(\Gamma^{\infty}(L)[[\lambda]], \bullet^{\prime}, \bullet\right)$ for the time being and to plug in the symplectomorphism $\Psi$ later on by hand.

Let $L$ be a line bundle and let $\mathcal{L}=\mathcal{L}[[\lambda]]$ be a deformation of the sections $\mathcal{L}=\Gamma^{\infty}(L)$ into an equivalence bimodule for two star products $\star$ and $\star^{\prime}$. Assume furthermore, that the first order terms $C_{1}$ and $C_{1}^{\prime}$ of $\star$ and $\star^{\prime}$ as in (3.1) coincide. For example, Fedosov star products always have 
the same first order term given by $\frac{\mathrm{i}}{2}$ times the Poisson bracket. From [5, Prop. 4.3] we know that the difference of the first order terms of the deformed module structures gives rise to a connection on $L$, called the induced connection. For Hamiltonian vector fields $X_{f}$ with $f \in C^{\infty}(M)$ it is defined by

$$
\nabla_{X_{f}}^{L}(s)=\mathrm{i} R_{1}^{\prime}(f, s)-\mathrm{i} R_{1}(s, f),
$$

and by $C^{\infty}(M)$-linear expansion this gives a well-defined connection since the Hamiltonian vector fields span the tangent spaces at every point. Note that in the Poisson case we would only obtain a contravariant connection. If we pass to an equivalent bimodule deformation by an equivalence transformation this connection does not change, see [8, Prop. 4.17].

If $\mathcal{L}$ was endowed with inner products defining a *-equivalence bimodule for the two star products, the classical limit yields in addition a pseudo Hermitian fiber metric on the line bundle in the classical limit. Indeed, one defines the classical limit of the inner product $\boldsymbol{h}$ by

$$
h\left(s, s^{\prime}\right)=\left.\boldsymbol{h}\left(\boldsymbol{s}, \boldsymbol{s}^{\prime}\right)\right|_{\lambda=0},
$$

where $s, s^{\prime} \in \mathcal{L}$ are arbitrary representatives of $s, s^{\prime} \in \mathcal{L}$, respectively. It is well known that this gives a well-defined full inner product for the classical limit as we are in the unital situation and $\boldsymbol{h}$ was non-degenerate and full already.

Remark 5.2 Of course, if $M$ is connected, then a pseudo Hermitian metric $h$ on a line bundle is necessarily either positive or negative definite. The issue of interesting signatures does not arise in this situation.

Moreover, if $\boldsymbol{h}$ was completely positive then also its classical limit is completely positive and hence a Hermitian fiber metric, see the discussion in [9]. Moreover, we note that the inner product $\boldsymbol{h}$ as well as its classical limit $h=\operatorname{cl}(\boldsymbol{h})$ do not change when we move from $\mathcal{L}$ to ${ }^{\Psi} \mathcal{L}$ : indeed, the twisting with the symplectomorphism modifies only the left module structure. Thus we can absorb the symplectomorphism from the beginning and assume $\mathcal{L}=\Gamma^{\infty}(L)[[\lambda]]$ also in this situation. This induces an inner product $\boldsymbol{h}$ on $\Gamma^{\infty}(L)[[\lambda]]$ which can be written as

$$
\boldsymbol{h}=\sum_{r=0}^{\infty} \lambda^{r} h_{r}
$$

with sesquilinear maps $h_{r}: \Gamma^{\infty}(L) \times \Gamma^{\infty}(L) \longrightarrow C^{\infty}(M)$. In this case the classical limit of $\boldsymbol{h}$ is, of course, given by $h_{0}$.

The following simple lemma clarifies the compatibility between the classical limit of the inner product and the induced connection:

Lemma 5.3 Let $\mathcal{L}=\left(\Gamma^{\infty}(L)[[\lambda]], \bullet, \bullet^{\prime}, \boldsymbol{h}\right)$ be $a^{*}$-equivalence bimodule for the Hermitian star products $\star$ and $\star^{\prime}$ deforming a pseudo Hermitian line bundle $\left(L, h_{0}\right)$. Assume furthermore $C_{1}=C_{1}^{\prime}$ for the first order terms of the star products. Then the induced connection $\nabla^{L}$ on $L$ is compatible with the induced pseudo Hermitian fiber metric $h_{0}$.

Proof. The proof is a matter of straightforward computation. Let $s, s^{\prime} \in \Gamma^{\infty}(L)$ and $f \in C^{\infty}(M)$. The semiclassical limit of $\boldsymbol{h}(s, b \bullet f)=\boldsymbol{h}(s, b) \star f$ yields

$$
h_{1}\left(s, s^{\prime} f\right)+h_{0}\left(s, R_{1}\left(s^{\prime}, f\right)\right)=h_{1}\left(s, s^{\prime}\right) f+C_{1}\left(h_{0}\left(s, s^{\prime}\right), f\right) .
$$

From $\boldsymbol{h}\left(s \bullet f, s^{\prime}\right)=\bar{f} \star \boldsymbol{h}\left(s, s^{\prime}\right)$ we get

$$
h_{1}\left(s f, s^{\prime}\right)+h_{0}\left(R_{1}(s, f), s^{\prime}\right)=\bar{f} h_{1}\left(s, s^{\prime}\right)+C_{1}\left(\bar{f}, h_{0}\left(s, s^{\prime}\right)\right),
$$


and $\boldsymbol{h}\left(f \bullet^{\prime} s, s^{\prime}\right)=\boldsymbol{h}\left(s, \bar{f} \bullet \bullet^{\prime}\right)$ gives

$$
h_{1}\left(f s, s^{\prime}\right)+h_{0}\left(R_{1}^{\prime}(f, s), s^{\prime}\right)=h_{1}\left(s, \bar{f} s^{\prime}\right)+h_{0}\left(s, R_{1}^{\prime}\left(\bar{f}, s^{\prime}\right)\right) .
$$

Taking $f=\bar{f}$ and substracting $(*)$ from $(* *)$ yields

$$
h_{1}\left(s f, s^{\prime}\right)-h_{1}\left(s, f s^{\prime}\right)=h_{0}\left(s, R_{1}\left(s^{\prime}, f\right)\right)-h_{0}\left(R_{1}(s, f), s^{\prime}\right)+C_{1}\left(f, h_{0}\left(s, s^{\prime}\right)\right)-C_{1}\left(h_{0}\left(s, s^{\prime}\right), f\right),
$$

whereas $(* * *)$ shows, that

$$
h_{1}\left(s f, s^{\prime}\right)-h_{1}\left(s, f s^{\prime}\right)=h_{0}\left(s, R_{1}^{\prime}\left(f, s^{\prime}\right)\right)-h_{0}\left(R_{1}^{\prime}(f, s), s^{\prime}\right) .
$$

Together with $C_{1}(f, g)-C_{1}(g, f)=\mathrm{i}\{f, g\}$, we finally conclude that

$$
\mathcal{L}_{X_{f}}\left(h_{0}\left(s, s^{\prime}\right)\right)=\left\{h_{0}\left(s, s^{\prime}\right), f\right\}=h_{0}\left(\nabla_{X_{f}}^{L} s, s^{\prime}\right)+h_{0}\left(s, \nabla_{X_{f}}^{L} s^{\prime}\right) .
$$

Again, since the Hamiltonian vector fields span the tangent space at each point, this is enough to show the claim.

After this preparation we want to discuss the semiclassical limit of the $\mathfrak{g}$-symmetry. The following observation is now a trivial computation:

Lemma 5.4 Let $\mathcal{L}$ be a $\mathfrak{g}$-equivariant equivalence bimodule. For $\xi \in \mathfrak{g}$ and $s \in \mathcal{L}=\operatorname{cl}(\mathcal{L})$,

$$
\rho(\xi) s=\operatorname{cl}(\xi \triangleright s)
$$

defines a lift of the classical $\mathfrak{g}$-action on $C^{\infty}(M)$ to $\mathcal{L}$ where $\boldsymbol{s} \in \mathcal{L}$ is any representative of $s$. This turns $\mathcal{L}$ into a $\mathfrak{U}_{\mathbb{C}}(\mathfrak{g})$-equivariant equivalence bimodule for $C^{\infty}(M)$.

Lemma 5.5 Let $\mathcal{L}$ be a $\mathfrak{g}$-equivariant equivalence bimodule and let $\Psi$ be the unique symplectomorphism which makes the classical limit of ${ }^{\Psi} \mathcal{L}$ a static equivalence bimodule. Then $\Psi$ is $\mathfrak{g}$-equivariant.

Proof. We know that the classical limit $\operatorname{cl}(\mathcal{L})$ of $\mathcal{L}$ is a $\mathcal{U}_{\mathbb{C}}(\mathfrak{g})$-equivariant equivalence bimodule for $C^{\infty}(M)$. By Proposition 4.11 we know that $\operatorname{cl}(\mathcal{L})$ splits uniquely into an equivariant automorphism $\Psi^{*}$ of $C^{\infty}(M)$ and an equivariant static equivalence bimodule. This automorphism $\Psi^{*}$ is the pullback of the symplectomorphism we are looking for.

Lemma 5.6 Let $\mathcal{L}=\left(\Gamma^{\infty}(L)[[\lambda]], \bullet, \bullet '\right)$ be a $\mathfrak{g}$-equivariant equivalence bimodule for the $\mathfrak{g}$-invariant star products $\star$ and $\star^{\prime}$ deforming a line bundle L. Assume $C_{1}=C_{1}^{\prime}$. Then the induced connection $\nabla^{L}$ is $\mathfrak{g}$-invariant with respect to the classical limit action (5.5).

Proof. Let $\xi \in \mathfrak{g}, f \in C^{\infty}(M)$, and $s \in \Gamma^{\infty}(L)$. Evaluating the equation $\xi \triangleright(s \bullet f)=(\xi \triangleright s) \bullet f+$ $s \bullet(\xi \triangleright f)$ in order $\lambda$ gives $\rho(\xi) R_{1}(s, f)=R_{1}(\rho(\xi) s, f)+R_{1}(s, \rho(\xi) f)$. Together with the analogous equation for the left module structure we get

$$
\rho(\xi)\left(\nabla_{X_{f}}^{L} s\right)=\nabla_{\rho(\xi) X_{f}}^{L} s+\nabla_{X_{f}}^{L}(\rho(\xi) s) .
$$

As usual, this yields the desired compatibility for all vector fields.

Lemma 5.7 Let $\mathcal{L}=\left(\Gamma^{\infty}(L)[[\lambda]], \bullet, \bullet^{\prime}, \boldsymbol{h}\right)$ be a $\mathfrak{g}$-equivariant ${ }^{*}$-equivalence bimodule for the $\mathfrak{g}$ invariant Hermitian star products $\star$ and $\star^{\prime}$ deforming a pseudo Hermitian line bundle $\left(L, h_{0}\right)$. Then the pseudo Hermitian fiber metric $h_{0}$ is $\mathfrak{g}$-invariant with respect to the classical limit action (5.5). 
Proof. First note that (2.2) means $\xi \triangleright \boldsymbol{h}\left(s, s^{\prime}\right)=\boldsymbol{h}\left(\xi \triangleright s, s^{\prime}\right)+\boldsymbol{h}\left(s, \xi \triangleright s^{\prime}\right)$ for $\xi \in \mathfrak{g}$ and $s, s^{\prime} \in$ $\Gamma^{\infty}(L)$. Evaluating this in zeroth order gives immediately the result $\rho(\xi) h_{0}\left(s, s^{\prime}\right)=h_{0}\left(\rho(\xi) s, s^{\prime}\right)+$ $h_{0}\left(s, \rho(\xi) s^{\prime}\right)$. But this is the desired invariance of $h_{0}$.

We can now collect the single results into the following theorem which clarifies the semiclassical limit of $\mathfrak{g}$-equivariant equivalence bimodules completely:

Theorem 5.8 (Semiclassical limit) Assume that on $M$ we have a $\mathfrak{g}$-invariant connection. Let $\star$ and $\star^{\prime}$ be two $\mathfrak{g}$-invariant star products on $M$.

i.) Assume $\star$ and $\star^{\prime}$ are $\mathfrak{g}$-equivariantly Morita equivalent via a $\mathfrak{g}$-equivariant equivalence bimodule $\mathcal{L}$. Then there exists a unique $\mathfrak{g}$-equivariant symplectomorphism $\Psi$, a line bundle $L$ unique up to isomorphism with a lift of the $\mathfrak{g}$-action, and a $\mathfrak{g}$-invariant connection $\nabla^{L}$ on $L$ such that $\mathcal{L}$ is isomorphic to the $\Psi$-twist of a bimodule deformation $\left(\Gamma^{\infty}(L)[[\lambda]], \bullet, \bullet^{\prime \prime}\right)$ of the sections of the line bundle with respect to $\star$ and $\star^{\prime \prime}$ where $\star^{\prime \prime}$ is $\mathfrak{g}$-equivariantly equivalent to $\Psi^{*}\left(\star^{\prime}\right)$ such that $\star^{\prime \prime}$ has the same first order term than $\star$. Finally, the connection is obtained as first order difference of the deformed bimodule deformations $\bullet$ and $\bullet$ ".

ii.) If in addition $\star$ and $\star^{\prime}$ are Hermitian and $\mathcal{L}$ is even a $\mathfrak{g}$-equivariant ${ }^{*}$-equivalence bimodule then in addition we have a $\mathfrak{g}$-invariant pseudo Hermitian metric $h_{0}$ on L, with $\nabla^{L}$ being metric, such that the $\left(C^{\infty}(M)[[\lambda]], \star\right)$-valued inner product is a deformation of $h_{0}$.

iii.) If furthermore $\mathcal{L}$ is even a $\mathfrak{g}$-equivariant strong equivalence bimodule then $h_{0}$ is Hermitian.

Proof. Let $\mathcal{L}$ be the $\mathfrak{g}$-equivariant equivalence bimodule for $\star^{\prime}$ acting from the left and $\star$ from the right. First we apply Proposition 5.1 to split off the symplectomorphism $\Psi$ by passing from $\star^{\prime}$ to $\Psi^{*}\left(\star^{\prime}\right)$. By Lemma 5.5, $\Psi$ is necessarily $\mathfrak{g}$-equivariant so $\Psi^{*}\left(\star^{\prime}\right)$ is still a $\mathfrak{g}$-invariant star product. The twisted bimodule is now a $\mathfrak{g}$-equivariant equivalence bimodule for $\Psi^{*}\left(\star^{\prime}\right)$ and $\star$ such that its classical limit is static. Next we can pass from $\Psi^{*}\left(\star^{\prime}\right)$ to an equivalent star product via a $\mathfrak{g}$-equivariant equivalence transformation in such a way that the new star product $\star^{\prime \prime}$ has the same first order term as $\star$. Here we need the $\mathfrak{g}$-invariant connection on $M$ to achieve this in a $\mathfrak{g}$-equivariant way: indeed, having a $\mathfrak{g}$-invariant connection, we can write the $\mathfrak{g}$-invariant Hochschild 2-coboundary $C_{1}-\Psi^{*}\left(C_{1}^{\prime}\right)$ as Hochschild $\delta$ of some $\mathfrak{g}$-invariant differential operator $D_{1}$ according to [4, Prop. 2.1]. Then exponentiating $D_{1}$ gives a g-equivariant equivalence transformation from $\Psi^{*}\left(\star^{\prime}\right)$ to $\star^{\prime \prime}$ where now $\star^{\prime \prime}$ has the same first order as $\star$. Correspondingly, we can transport the equivalence bimodule along. This results in a $\left(\star^{\prime \prime}, \star\right)$-equivalence bimodule which is isomorphic to $\Gamma^{\infty}(L)[[\lambda]]$ endowed with deformed bimodule multiplications $\bullet$ and $\bullet$ " where $L$ is a line bundle uniquely determined up to isomorphism. Moreover, by Lemma 5.4 the line bundle carries an action of $\mathfrak{g}$ which is a lift of the action on $M$. With Lemma 5.3 we get the connection $\nabla^{L}$ which is $\mathfrak{g}$-invariant by Lemma 5.6. This shows the ring-theoretic part. For the case of Hermitian star products and *-equivalences we use Lemma 5.3 and Lemma 5.7 to conclude that the induced pseudo Hermitian metric has the desired properties. Finally, the last part is clear.

Remark 5.9 If we are only interested in star products where the first order term is $\frac{\mathrm{i}}{2}\{\cdot, \cdot\}$ (and not just its antisymmetric part) then the extra assumption of an invariant connection on $M$ becomes superfluous. Also, if we are not interested in the invariant connection $\nabla^{L}$ on $L$ we can omit this assumption.

\subsection{Characterization of equivariant Morita equivalent star products}

Now we look for necessary and sufficient conditions for equivariant Morita equivalence of star products: in some sense we want to investigate how the conditions in the semiclassical limit are 
already sufficient to guarantee the corresponding equivalence also in the full "quantum" situation.

It is well known that two star products $\star$ and $\star^{\prime}$ over $(M, \omega)$ are Morita equivalent if and only if there is a line bundle $L \longrightarrow M$ and a symplectomorphism $\Psi: M \longrightarrow M$ such that $\Psi^{*} c\left(\star^{\prime}\right)-c(\star)=2 \pi \mathrm{i} c_{1}(L)$, see [7]. As before, $\Psi^{*}\left(\star^{\prime}\right)$ denotes the star product $\star^{\prime}$ twisted with $\Psi$ and $c(\star)$ is the characteristic class of $\star$. Finally, $c_{1}(L) \in \mathrm{H}_{\mathrm{dR}}^{2}(M, \mathbb{Z})$ is the Chern class of $L$ viewed as class in the deRham cohomology. Choosing a covariant derivative $\nabla^{L}$ with curvature two-from $R^{L} \in \Omega^{2}(M)$ for $L$, we have $\left[R^{L}\right]=2 \pi \mathrm{i} c_{1}(L) \in \mathrm{H}_{\mathrm{dR}}^{2}(M, \mathbb{C})$. Note that this is not the Chern class in the second integral cohomology of $M$ but it's image in the deRham cohomology, so possible torsion bundles will have trivial class $c_{1}(L)=0$ in this version.

First we want to assume that there is already an action of $\mathfrak{g}$ on a given line bundle $L$. Then we can modify the Fedosov construction for the bimodule $\Gamma^{\infty}(L)$ to the $\mathfrak{g}$-invariant case, as is done in [15, 21] for the case of a Lie group action on $L \longrightarrow M$.

Proposition 5.10 (Invariant Fedosov construction) Let $L \longrightarrow M$ be a line bundle over a symplectic manifold $M$. Suppose $\mathfrak{g}$ acts on $M$ via symplectic vector fields and there is a lift of this action to $L$. Moreover, suppose there is a $\mathfrak{g}$-invariant symplectic connection on $M, \Omega \in$ $\lambda \Omega^{2}(M, \mathbb{C})[[\lambda]]$ is a formal series of $\mathfrak{g}$-invariant closed two-forms, and $L$ allows for a $\mathfrak{g}$-invariant connection $\nabla^{L}$.

i.) The Fedosov construction leads to a $\mathfrak{g}$-equivariant equivalence bimodule $\left(\Gamma^{\infty}(L)[[\lambda]], \bullet, \bullet^{\prime}\right)$ for the $\mathfrak{g}$-invariant Fedosov star products $\star_{\nabla, \Omega}$ and $\star^{\prime}=\star_{\nabla, \Omega^{\prime}}$ with $\Omega^{\prime}=\Omega+\mathrm{i} \lambda R^{L}$, acting from the left. Hence the $\mathfrak{g}$-invariant characteristic classes are $c^{\mathfrak{g}}(\star \nabla, \Omega)=\frac{1}{\mathrm{i} \lambda}[\omega+\Omega]$ and $c^{\mathfrak{g}}\left(\star^{\prime}\right)=$ $c^{\mathfrak{g}}(\star)+2 \pi \mathrm{i} c_{1}(L)$.

ii.) If in addition $\Omega$ is real and if there exists a $\mathfrak{g}$-invariant pseudo Hermitian metric $h_{0}$ on $L$ such that $\nabla^{L}$ is metric then $\star$ and $\star^{\prime}$ are Hermitian and $h_{0}$ can be deformed into a $\mathfrak{g}$-invariant $\left(C^{\infty}(M)[[\lambda]], \star\right)$-valued inner product making the above bimodule even a $\mathfrak{g}$-equivariant ${ }^{*}$ equivalence bimodule.

iii.) In in addition $h_{0}$ is Hermitian then the resulting inner products are completely positive and the bimodule is a $\mathfrak{g}$-equivariant strong equivalence bimodule.

Proof. The functoriality of the Fedosov construction [15, 21] give this result immediately.

Lemma 5.11 Let $L \longrightarrow M$ be a line bundle such that $c_{1}(L) \in \mathrm{H}_{\mathrm{dR}}^{2}(M, \mathbb{Z})$ is in the image of the combined map of (3.20) after (3.8). For every Hermitian fiber metric $h_{0}$ on $L$ there is a metric connection $\nabla^{L}$ and a lifting of the $\mathfrak{g}$-action to $L$ such that $h_{0}$ and $\nabla^{L}$ are invariant.

From this folklore statement, see e.g. [18,19], we immediately obtain the characterization of equivariant (strong) Morita equivalence proving Theorem 1.1

Proof of Theorem 1.1. If they are $\mathfrak{g}$-equivariantly (strongly) Morita equivalent then our considerations from the semiclassical limit in Theorem 5.8 show that their characteristic classes combine into the $\mathfrak{g}$-invariant Chern class of a line bundle $L$ with $\mathfrak{g}$-action, $2 \pi \mathrm{i} c_{1}(L)=\Psi^{*} c\left(\star^{\prime}\right)-c(\star) \in$ $2 \pi \mathrm{iH}_{\mathrm{dR}}(M, \mathbb{Z})$. Without $\mathfrak{g}$-invariance, this is the original characterization from [7]. Moreover, we know that on $L$ there is a $\mathfrak{g}$-invariant connection $\nabla^{L}$ and hence its curvature $R^{L}$ is a $\mathfrak{g}$-invariant representative of $2 \pi \mathrm{i} c_{1}(L)$. Now define $J^{L}(\xi) \in \operatorname{End}_{C^{\infty}(M)}\left(\Gamma^{\infty}(L)\right)=C^{\infty}(L)$ via $J^{L}(\xi)=-\rho(\xi)-\nabla_{X_{\xi}}^{L}$ where $\rho(\xi)$ is the action of $\xi \in \mathfrak{g}$ on $\Gamma^{\infty}(L)$. From the definition of $\rho(\xi)=-\mathcal{L}_{X_{\xi}}$ on functions it follows that $J^{L}(\xi)$ is indeed $C^{\infty}(M)$-linear and hence a function itself. Since $\nabla^{L}$ is invariant and since $\rho(\cdot)$ is a Lie algebra action by module derivations it follows by a straightforward computation that $J^{L}$ extends $R^{L}$ to an equivariant two-form as wanted. 
Conversely, we first note that every $\mathfrak{g}$-invariant (Hermitian) star product is $\mathfrak{g}$-equivariantly equivalent (with a real equivalence transformation since a $\mathfrak{g}$-equivariant equivalence can be made a *-equivalence according to [23, Exercise 7.6] without destroying the $\mathfrak{g}$-equivariance) to a Fedosov star product (with real $\Omega$ ). The first condition on the characteristic classes shows that the star products are Morita equivalent and the corresponding line bundle can be used to build the equivalence bimodule. Note that $\Psi^{*} c\left(\star^{\prime}\right)-c(\star)$ is necessarily concentrated in zeroth order of $\lambda$ only. By Lemma 5.11 we have a lift to this line bundle, a $\mathfrak{g}$-invariant Hermitian metric, and a $\mathfrak{g}$-invariant metric connection. Then the Fedosov construction from Proposition 5.10 ensures that the corresponding Fedosov star products and hence also the original star products are g-equivariantly (strongly) Morita equivalent.

\subsection{The role of quantum momentum maps}

We conclude this section with some remarks on the role of quantum momentum maps.

To apply our lifting statements for general Hopf algebra actions from Theorem 4.7 we have to use the Hopf ${ }^{*}$-algebra $\mathcal{U}_{\lambda}(\mathfrak{g})$ for star products with quantum momentum map instead of $\mathcal{U}_{\mathbb{C}}(\mathfrak{g})$. In this case, the action on $C^{\infty}(M)[[\lambda]]$ of $\mathcal{U}_{\lambda}(\mathfrak{g})$ is given by (3.12) and therefore starts in first order of $\lambda$ but not in the zeroth order. We can always pass from a $\mathcal{U}_{\mathbb{C}}(\mathfrak{g})$-action to a corresponding action of $\mathcal{U}_{\lambda}(\mathfrak{g})$ in the following way: Let $\mathcal{L}$ be a $\mathbb{C}[[\lambda]]$-module with a left $\mathcal{U}_{\mathbb{C}}(\mathfrak{g})$-action $\rho$ on it. Then

$$
\xi \triangleright x=\mathrm{i} \lambda \rho(\xi) x
$$

for $\xi \in \mathfrak{g}$ and $x \in \mathcal{L}$ extends uniquely to an action of $\mathcal{U}_{\lambda}(\mathfrak{g})$ on $\mathcal{L}$. However, not every $\mathcal{U}_{\lambda}(\mathfrak{g})$-action is of that form. The reason is that all operators $\xi \triangleright$. have to vanish for $\lambda=0$, i.e. in the classical limit, for $\xi \in \mathfrak{g}$. However, for the lift according to Theorem 4.7 to equivalence bimodules this is automatically the case:

Lemma 5.12 Let two $\mathfrak{g}$-invariant star products $\star, \star^{\prime}$ be Morita equivalent with an equivalence bimodule $\mathcal{L}$, and let $\Psi$ be the symplectomorphism such that ${ }^{\Psi} \operatorname{cl}(\mathcal{L})$ is static. Assume $\star$ and $\star^{\prime}$ have quantum momentum maps $J$ and $J^{\prime}$. For the canonical lift (4.4) of the $\mathcal{U}_{\lambda}(\mathfrak{g})$-action to $\mathcal{L}$ there exists a unique action $\rho$ of $\mathcal{U}_{\mathbb{C}}(\mathfrak{g})$ such that $\triangleright$ is of the form (5.6) iff the induced symplectomorphism $\Psi$ satisfies $\Psi^{*} J_{0}^{\prime}=J_{0}$.

Proof. We know that $\mathcal{L} \cong \Psi \Gamma^{\infty}(L)[[\lambda]]$ as $\mathbb{C}[[\lambda]]$-modules which induces deformed module structures $\bullet$ and $\bullet$ on ${ }^{\Psi} \Gamma^{\infty}(L)[[\lambda]]$. The lifted action (4.4) is now given by

$$
\xi \triangleright s=J^{\prime}(\xi) \bullet ' s-s \bullet J(\xi)
$$

for $s \in \Gamma^{\infty}(L)[[\lambda]]$ and $\xi \in \mathfrak{g}$. The classical limit of $(*)$ is $\Psi^{*}\left(J_{0}^{\prime}(\xi)\right) s-s J_{0}(\xi)$ which is zero iff $\Psi^{*} J_{0}^{\prime}(\xi)=J_{0}(\xi)$ for all $\xi \in \mathfrak{g}$. It is clear that $(*)$ is of the form (5.6) iff it vanishes in the zeroth order.

Since both classical limits $J_{0}$ and $J_{0}^{\prime}$ are classical momentum maps it follows that a symplectomorphism $\Psi$ with $\Psi^{*} J_{0}^{\prime}=J_{0}$ is $\mathfrak{g}$-equivariant. Conversely, if $\Psi$ is $\mathfrak{g}$-equivariant then $\Psi^{*} J_{0}^{\prime}$ is still a classical momentum map and hence differs from $J_{0}$ only by a cocycle $c$ in $Z^{1}(\mathfrak{g}, \mathbb{C})$. Then the lifted $\mathcal{U}_{\lambda}(\mathfrak{g})$-action in zeroth order is given by $\xi \triangleright s=c_{\xi} s+\cdots$ which does not vanish. However, in this case we can modify the $\mathfrak{U}_{\lambda}(\mathfrak{g})$-action by precisely this cocycle. This results in a $\mathfrak{U}_{\lambda}(\mathfrak{g})$-equivalence bimodule with action

$$
\xi \triangleright^{\prime} s=J^{\prime}(\xi) \bullet ' s-s \bullet J(\xi)-c_{\xi} s
$$

for $\xi \in \mathfrak{g}$ and $s \in \Gamma^{\infty}(L)[[\lambda]]$. By $c_{[\xi, \eta]}=0$ this clearly extends to a $\mathcal{U}_{\lambda}(\mathfrak{g})$-action. Since $c_{\xi}$ is a constant we still have compatibility with the bimodule structure. This way, the action $\triangleright^{\prime}$ is now of the form (5.6) with an action $\rho$ of $\mathcal{U}_{\mathbb{C}}(\mathfrak{g})$. We summarize this in the following lemma: 
Lemma 5.13 Let $\star$ and $\star^{\prime}$ be $\mathfrak{g}$-invariant star products with quantum momentum maps $J$ and $J^{\prime}$. Let $\mathcal{L}$ be an equivalence bimodule with induced symplectomorphism $\Psi$. If $\Psi$ is $\mathfrak{g}$-equivariant then $\star$ and $\star^{\prime}$ are $\mathfrak{g}$-equivariantly Morita equivalent.

Proof. Indeed, by the above considerations the cocycle $c_{\xi}=\Psi^{*} J_{0}^{\prime}(\xi)-J_{0}(\xi)$ can be used to modify the lifted action (4.4) to obtain an action of the form (5.6). Thus $\mathcal{L}$ with this action is a $\mathcal{U}_{\mathbb{C}}(\mathfrak{g})$ equivariant equivalence bimodule for $\star^{\prime}$ and $\star$.

In this situation where we have such an equivalence bimodule with $\mathfrak{g}$-invariant symplectomorphism $\Psi$ we see that also $\Psi^{*}\left(\star^{\prime}\right)$ is a $\mathfrak{g}$-invariant star product with quantum momentum map $\Psi^{*} J^{\prime}$. Moreover, we see that $c^{\mathfrak{g}}\left(\Psi^{*}\left(\star^{\prime}\right)\right)=\Psi^{*} c^{\mathfrak{g}}\left(\star^{\prime}\right)$ since $\Psi$ is $\mathfrak{g}$-equivariant. By Morita equivalence we have that $\Psi^{*} c^{\mathfrak{g}}\left(\star^{\prime}\right)-c^{\mathfrak{g}}(\star)$ maps to the $2 \pi \mathrm{i}$-integral deRham cohomology, i.e. $\Psi^{*} c^{\mathfrak{g}}\left(\star^{\prime}\right)-c^{\mathfrak{g}}(\star) \in 2 \pi \mathrm{i} H_{\mathrm{dR}}^{2}(M, \mathbb{Z})$. Finally, since the star products $\Psi^{*}\left(\star^{\prime}\right)$ and $\star$ have quantum momentum maps it is clear that their invariant characteristic classes are in the image of (3.20) by Proposition 3.3. But then trivially the difference $\Psi^{*} c^{\mathfrak{g}}\left(\star^{\prime}\right)-c^{\mathfrak{g}}(\star)$ is in the image as well. This way, we recover the general statement of Theorem 1.1 for star products with quantum momentum maps. Note that now the lifting part of Theorem 1.1 was now done using the general lifting construction from Theorem 4.7 .

Remark 5.14 In the case of strong Morita equivalence we can argue analogously, observing that for real momentum maps the above cocycle is real, too. Hence the inner products will not be influenced at all.

\section{References}

[1] ARA, P.: Morita equivalence for rings with involution. Alg. Rep. Theo. 2 (1999), 227-247.

[2] Bass, H.: Algebraic K-Theory. W. A. Benjamin, Inc., New York, Amsterdam, 1968.

[3] Bayen, F., Flato, M., Frønsdal, C., Lichnerowicz, A., Sternheimer, D.: Deformation Theory and Quantization. Ann. Phys. 111 (1978), 61-151.

[4] Bertelson, M., Bieliavsky, P., Gutt, S.: Parametrizing Equivalence Classes of Invariant Star Products. Lett. Math. Phys. 46 (1998), 339-345.

[5] Bursztyn, H.: Semiclassical geometry of quantum line bundles and Morita equivalence of star products. Int. Math. Res. Not. 2002.16 (2002), 821-846.

[6] Bursztyn, H., Dolgushev, V., Waldmann, S.: Morita equivalence and characteristic classes of star products. Preprint arXiv:0909:4259 (September 2009), 57 pages.

[7] Bursztyn, H., Waldmann, S.: The characteristic classes of Morita equivalent star products on symplectic manifolds. Commun. Math. Phys. 228 (2002), 103-121.

[8] Bursztyn, H., Waldmann, S.: Bimodule deformations, Picard groups and contravariant connections. KTheory 31 (2004), 1-37.

[9] Bursztyn, H., Waldmann, S.: Completely positive inner products and strong Morita equivalence. Pacific J. Math. 222 (2005), 201-236.

[10] Dolgushev, V. A.: Covariant and equivariant formality theorems. Adv. Math. 191 (2005), 147-177.

[11] Fedosov, B. V.: A Simple Geometrical Construction of Deformation Quantization. J. Diff. Geom. 40 (1994), 213-238.

[12] Guillemin, V. W., Sternberg, S.: Supersymmetry and Equivariant de Rham Theory. Springer-Verlag, Berlin, Heidelberg, New York, 1999.

[13] Gutt, S., Rawnsley, J.: Equivalence of star products on a symplectic manifold; an introduction to Deligne's Čech cohomology classes. J. Geom. Phys. 29 (1999), 347-392.

[14] Gutt, S., Rawnsley, J.: Natural Star Products on Symplectic Manifolds and Quantum Moment Maps. Lett. Math. Phys. 66 (2003), 123-139. 
[15] Jansen, S.: H-Äquivariante Morita-Äquivalenz und Deformationsquantisierung. PhD thesis, Fakultät für Mathematik und Physik, Physikalisches Institut, Albert-Ludwigs-Universität, Freiburg, November 2006.

[16] Jansen, S., Waldmann, S.: The H-covariant strong Picard groupoid. J. Pure Appl. Alg. 205 (2006), 542-598.

[17] Jurčo, B., Schupp, P., Wess, J.: Noncommutative Line Bundles and Morita Equivalence. Lett. Math. Phys. 61 (2002), 171-186.

[18] Kostant, B.: Quantization and Unitary Representation. Part I: Prequantization. In: TAAm, C. T. (Eds.): Lectures in Modern Analysis and Application, vol. 170 in Lecture Notes in Mathematics, 87-208. Springer-Verlag, Berlin, 1970.

[19] Lashof, R.: Equivariant Prequantization. In: Dazord, P., Weinstein, A. (Eds.): Symplectic Geometry, Groupoids, and Integrable Systems, vol. 20 in Mathematical Sciences Research Institute Publications, $193-207$. Springer-Verlag, New York, Berlin, Heidelberg, 1991.

[20] Müller-Bahns, M. F., Neumaier, N.: Some remarks on $\mathfrak{g}$-invariant Fedosov star products and quantum momentum mappings. J. Geom. Phys. 50 (2004), 257-272.

[21] Waldmann, S.: Morita equivalence of Fedosov star products and deformed Hermitian vector bundles. Lett. Math. Phys. 60 (2002), 157-170.

[22] Waldmann, S.: The covariant Picard groupoid in differential geometry. Int. J. Geom. Methods Mod. Phys. 3.3 (2006), 641-654.

[23] Waldmann, S.: Poisson-Geometrie und Deformationsquantisierung. Eine Einführung. Springer-Verlag, Heidelberg, Berlin, New York, 2007. 